\title{
Characterizations and Infinite Divisibility of Certain Recently Introduced Distributions III
}

\author{
G.G. Hamedani ${ }^{1}$ \\ ${ }^{1}$ Department of Mathematics, Statistics and Computer Science, Marquette University \\ Correspondence: G.G. Hamedani, Department of Mathematics, Statistics and Computer Science, Marquette University, \\ Milwaukee, WI 53201-1881.
}

\author{
Received: August 22, 2017 Accepted: September 13, 2017 Online Published: November 10, 2017 \\ doi:10.5539/ijsp.v7n1p39 \\ URL: https://doi.org/10.5539/ijsp.v7n1p39
}

\begin{abstract}
Certain characterizations of recently proposed univariate continuous distributions are presented in different directions. This work may be a source of preventing reinventing and duplicating the existing distributions and calling them newly proposed distributions.
\end{abstract}

Keywords: Continuous univariate distributions; Hazard function; Reverse hazard function; Infinitely divisible distributions; Characterizations.

\section{Introduction}

This work is a continuation of our previous works (Hamedani and Safavimanesh, 2016) and (Hamedani 2017) on characterizations and infinite divisibility of distributions introduced in 2016-2017. In designing a stochastic model for a particular modeling problem, an investigator will be vitally interested to know if their model fits the requirements of a specific underlying probability distribution. To this end, the investigator will rely on the characterizations of the selected distribution. Thus, the problem of characterizing a distribution is an important problem in various fields and has recently attracted the attention of many researchers. Consequently, various characterization results have been reported in the literature. These characterizations have been established in many different directions. This work deals with various characterizations of the following distributions: 1) Extension of the Generalized Linear Failure Rate (EGLFR) distribution of Kazemi et al.; 2) McDonald Gompertz (McG) distribution of Roozegar et al.; 3) Kumaraswamy-Transmuted Exponentiated Modified Weibull (Kw-TEMW) distribution of Al-babtain et al.; 4) Lomax-Exponential (LE) distribution of Hami Golzar et al.; 5) Truncated Exponential Exponential (TEE) distribution of Mahdavi and Silva; 6) Gompertz-G (Go-G) family of distributions of Alizadeh et al.; 7) Type I Half-Logistics (TIHL-G) family of distributions of Cordeiro et al.; 8) Transmuted Geometric-Weibull (TGW) distribution of Nofal et al.; 9) Adjusted Log-Logistic Generalized Exponential (ALLGE) distribution of Okorie et al.; 10) Type II Half Logistic (TIIHL-G) family of distributions of Hassan et al.; 11) Transmuted New Generalized Inverse Weibull (TNGIW) distribution of Khan et al.; 12) Poisson-G (Po-G) family of distributions of Abouelmagd et al.; 13) Generalized Transmuted Weibull (GTW) distribution of Nofal and El Gebaly; 14) Exponentiated Generalized-G Poisson (EGGP) family of distributions of Aryal and Yousof; 15) Beta Inverse Rayleigh (BIR) distribution of Leão et al.; 16) Beta Weibull Poisson (BWP) distribution of Percontini et al.; 17) Gamma Modified Weibull (GMW) distribution of Cordeiro et al.; 18) Transmuted Dagum (TD) distribution of Elbatal and Aryal; 19) Exponentiated Gompertz Generated (EGG) family of distributions of Cordeiro et al.; 20) New Extension of Power Lindley (NEPL) distribution of Alizadeh et al.; 21) Poisson-Weibull Regression (PWR) distribution of Vigas et al.; 22) Odd Log-Logistic Skew Normal (OLLSN) distribution of da Silva Braga et al.; 23) Extended Generalized Gamma Geometric (EGGG) distribution of Bortolini e al.; 24) Eponentiated Transmuted Quasi Lindley (ETQL) distribution of Preda; 25) Log-Kumaraswamy Generalized Gamma (LKGG) distribution of Pascoa et al.; 26) Exponentiated Reduced Kies (ERK) distribution of Kumar and Dharmaja; 27) Exponentiated Lomax (EL) distribution of Abdul-Moniem and Abdel_Hameed; 28) Another Extension of Exponential (AEE) distribution of Gómez et al.; 29) New Weighted Exponential (NWE) distribution of Kharazmi and Jabbari; 30) Exponentiated Inverse Weibull Geometric (EIWG) distribution of Chung et al.; 31) New Extension of the Exponential (NEE) distribution of Gómez et al.; 32) Exponentiated Transmuted Weibull (WTW) distribution of Ebraheim; 33) Generalizations of Weibull (GW) distribution of Jayakumar and Babu; 34) Exponentiated Nadarajah and Haghighi Exponential (ENHE) distribution of Abdul-Moniem; 35) Generalization of Weibull (GW) distribution of Babu; 36) Truncated Weibull-G (TW-G) distribution of Najarzadegan et al.; 37) Generalized Additive Weibull-G (GAW-G) family of distributions of Hassan et al.; 38) Two-parameter Weighted Exponential (TWE) distribution of Shakhatreh; 39) Generalized Weighted Weibull (GWW) distribution of Kharazmi; 40) Generalized Weighted Exponential (GWE) distribution of Kharazmi et al.; 41) Generalized Bilal (GB) distribution of Abd-Elrahman; . These characterizations are presented in different directions: $(i)$ based on the ratio of two truncated moments; (ii) in terms of the 
hazard function; (iii) in terms of the reverse (reversed) hazard function and (iv) based on the conditional expectation of certain function of the random variable. Note that $(i)$ can be employed also when the $c d f$ (cumulative distribution function) does not have a closed form. We would also like to mention that due to the nature of some of these 41 distributions, our chracterizations may be the only possible ones. In defining the above distributions we shall try to employ the same parameter notation as used by the original authors. We follow the same order as listed above.

1) The $c d f$ and $p d f$ (probability density function) of EGLFR are given, respectively, by

$$
F(x ; \alpha, \beta, a, b)=\left\{\begin{array}{l}
\left(1-\left(1-\beta\left(a x+\frac{b}{2} x^{2}\right)\right)^{1 / \beta}\right)^{\alpha}, \text { if } \beta \neq 0 \\
\left(1-\exp \left(-\left(a x+\frac{b}{2} x^{2}\right)\right)\right)^{\alpha}, \text { if } \beta=0
\end{array},\right.
$$

and

$$
f(x ; \alpha, \beta, a, b)= \begin{cases}\alpha(a+b x)(1-\beta z)^{\frac{1}{\beta}-1}\left(1-(1-\beta z)^{1 / \beta}\right)^{\alpha-1}, & \text { if } \beta \neq 0 \\ \alpha(a+b x) \exp (-z)(1-\exp (-z))^{\alpha-1}, & \text { if } \beta=0\end{cases}
$$

where $\alpha>0, \beta \in \mathbb{R}, a \geq 0$ and $b \geq 0$ (with $a+b>0$ ) are parameters, $z=\left(a x+\frac{b}{2} x^{2}\right.$ ) and $i$. If $\beta \leq 0$, then the support of the $c d f$ is $x \geq 0$.

ii. If $\beta>0$, then the support of the $c d f$ is $0 \leq x \leq \frac{1}{b} \sqrt{a^{2}+\frac{2 b}{\beta}}-\frac{a}{b}$.

iii.If $\beta>0$ and $b=0$, then the support of the $c d f$ is $0 \leq x \leq \frac{1}{a \beta}$.

2) The $c d f$ and $p d f$ of McG are given, respectively, by

$$
F(x ; a, b, c, \theta, \gamma)=\frac{1}{B(a / c, b)} \int_{0}^{\left[1-\exp \left(-\frac{\theta}{\gamma}\left(e^{\gamma x}-1\right)\right)\right]^{c}} w^{a-1}(1-w)^{b-1} d w, \quad x \geq 0,
$$

and

$$
\begin{gathered}
f(x ; a, b, c, \theta, \gamma)= \\
\frac{c \theta e^{\gamma x}}{B(a / c, b)} \exp \left(-\frac{\theta}{\gamma}\left(e^{\gamma x}-1\right)\right)\left[1-\exp \left(-\frac{\theta}{\gamma}\left(e^{\gamma x}-1\right)\right)\right]^{a c-1}\left\{1-\left[1-\exp \left(-\frac{\theta}{\gamma}\left(e^{\gamma x}-1\right)\right)\right]^{c}\right\}^{b-1}, \quad x>0,
\end{gathered}
$$

where $a, b, c, \theta, \gamma$ are all positive parameters.

3) The $c d f$ and $p d f$ of Kw-TEMW are given, respectively, by

$$
F(x ; \alpha, \beta, \theta, \gamma, a, b, \lambda)=1-\left\{1-\left[1-e^{-\left(\theta x+\gamma x^{\beta}\right)}\right]^{\alpha a}\left[1+\lambda-\lambda\left(1-e^{-\left(\theta x+\gamma x^{\beta}\right)}\right)^{\alpha}\right]^{a}\right\}^{b},
$$

$x \geq 0$ and

$$
\begin{aligned}
& f(x ; \alpha, \beta, \theta, \gamma, a, b, \lambda) \\
& =a b \alpha\left(\theta+\gamma \beta x^{\beta-1}\right) e^{-\left(\theta x+\gamma x^{\beta}\right)}\left[1+\lambda-2 \lambda\left(1-e^{-\left(\theta x+\gamma x^{\beta}\right)}\right)^{\alpha}\right] \times \\
& {\left[1-e^{-\left(\theta x+\gamma x^{\beta}\right)}\right]^{\alpha a-1}\left[1+\lambda-\lambda\left(1-e^{-\left(\theta x+\gamma x^{\beta}\right)}\right)^{\alpha}\right]^{a-1} \times} \\
& \left\{1-\left[1-e^{-\left(\theta x+\gamma x^{\beta}\right)}\right]^{\alpha a}\left[1+\lambda-\lambda\left(1-e^{-\left(\theta x+\gamma x^{\beta}\right)}\right)^{\alpha}\right]^{a}\right\}^{b-1}, x>0,
\end{aligned}
$$

where $\alpha, \beta, \theta, \gamma, a, b$ all positive and $\lambda(|\lambda| \leq 1)$ are parameters.

4) The $c d f$ and $p d f$ of LE are given, respectively, by

$$
F(x ; a, s, k)=1-\left(\frac{a}{e^{s x}+a-1}\right)^{k}, \quad x \geq 0,
$$

and

$$
f(x ; a, s, k)=\frac{s k a^{k} e^{s x}}{\left(e^{s x}+a-1\right)^{k+1}}, \quad x>0,
$$


where $a, s, k$ are positive parameters.

Remark 1. For $\alpha=1$ and $G_{1}(x)=\frac{e^{s x}-1}{e^{x x}+a-1}, x \geq 0$, the $c d f$ of NF (A New Family) of distributions of Alizadeh et al (2015) reduces to (7). The NF distribution has been characterized in the upcoming Research Monograph by Hamedani and Maadooliat (2017).

5) The $c d f$ and $p d f$ of TEE are given, respectively, by

$$
F(x ; \alpha, \lambda)=\frac{e^{\alpha}-e^{\alpha e^{-\lambda x}}}{e^{\alpha}-1}, \quad x \geq 0,
$$

and

$$
f(x ; \alpha, \lambda)=\frac{\alpha \lambda e^{-\lambda x} e^{\alpha e^{-\lambda x}}}{e^{\alpha}-1}, \quad x>0
$$

where $\alpha, \lambda$ are positive parameters.

Remark 2. For $\alpha=1$ and $G_{1}(x)=1-e^{-\lambda x}, x \geq 0$, the $c d f$ of EGP (The Exponentiated G Poisson) model of Gomes et al (2015) reduces to (9). The EGP distribution has been characterized in the upcoming Research Monograph by Hamedani and Maadooliat (2017).

6) The $c d f$ and $p d f$ of Go-G are given, respectively, by

$$
F(x ; \theta, \gamma, \eta)=1-e^{\frac{\theta}{\gamma}\left\{1-(\bar{G}(x ; \eta))^{-\gamma}\right\}}, \quad x \in \mathbb{R},
$$

and

$$
f(x ; \theta, \gamma, \eta)=\theta g(x ; \eta)(\bar{G}(x ; \eta))^{-\gamma-1} e^{\frac{\theta}{\gamma}\left\{1-(\bar{G}(x ; \eta))^{-\gamma}\right\}}, \quad x \in \mathbb{R},
$$

where $\theta, \gamma$ are positive parameters and $g(x ; \eta)$ and $G(x ; \eta)$ are $p d f$ and $c d f$ of the baseline distribution which depends on the vector parameter $\eta$.

7) The $c d f$ and $p d f$ of TIHL-G are given, respectively, by

$$
F(x ; \lambda)=\frac{1-(\bar{G}(x ; \eta))^{\lambda}}{1+(\bar{G}(x ; \eta))^{\lambda}}, \quad x \in \mathbb{R}
$$

and

$$
f(x ; \lambda)=\frac{2 \lambda g(x ; \eta)(\bar{G}(x ; \eta))^{\lambda-1}}{\left\{1+(\bar{G}(x ; \eta))^{\lambda}\right\}^{2}}, \quad x \in \mathbb{R}
$$

where $\lambda$ is a positive parameters and $g(x ; \eta)$ and $G(x ; \eta)$ are $p d f$ and $c d f$ of the baseline distribution which depends on the vector parameter $\eta$.

\section{Special TIHL-G distributions}

The following distributions are listed as sub-models of the TIHL-G distribution: The type I half-logistic normal; The type I half-logistic gamma and The type I half-logistic Fréchet.

Remark 3. For $\alpha=1$, the $c d f$ of EHL-G (The Exponentiated Half-Logistic Family) of distributions of Cordeiro et al (2014) reduces to (13). The EHL-G distribution has been characterized in the upcoming Research Monograph by Hamedani and Maadooliat (2017).

8) The $c d f$ and $p d f$ of TGW are given, respectively, by

$$
F(x ; \alpha, \beta, \theta, \lambda)=\frac{\theta\left(1-e^{-(\alpha x)^{\beta}}\right)}{1+(\theta-1)\left(1-e^{-(\alpha x)^{\beta}}\right)}\left\{1+\frac{\lambda e^{-(\alpha x)^{\beta}}}{1+(\theta-1)\left(1-e^{-(\alpha x)^{\beta}}\right)}\right\},
$$

$x \geq 0$ and

$$
f(x ; \alpha, \beta, \theta, \lambda)=\frac{\theta \beta \alpha^{\beta} x^{\beta-1}}{e^{(\alpha x)^{\beta}}\left[1+(\theta-1)\left(1-e^{-(\alpha x)^{\beta}}\right)\right]^{2}}\left\{1+\frac{\lambda e^{-(\alpha x)^{\beta}}}{1+(\theta-1)\left(1-e^{-(\alpha x)^{\beta}}\right)}\right\},
$$


$x>0$, where $\alpha, \beta, \theta$ positive and $\lambda(|\lambda| \leq 1)$ are parameters.

9) The $c d f$ and $p d f$ of ALLGE are given, respectively, by

$$
F(x ; \theta, \delta, \eta)=\frac{\left(\delta^{\theta}+1\right)\left(1-e^{-\eta x}\right)^{\theta}}{\delta^{\theta}+\left(1-e^{-\eta x}\right)^{\theta}}, \quad x \geq 0,
$$

and

$$
f(x ; \theta, \delta, \eta)=\frac{\left(\delta^{\theta}+1\right) \delta^{\theta} \theta \eta e^{-\eta x}\left(1-e^{-\eta x}\right)^{\theta-1}}{\left[\delta^{\theta}+\left(1-e^{-\eta x}\right)^{\theta}\right]^{2}}, \quad x>0,
$$

where $\theta, \delta, \eta$ are positive parameters.

10) The $c d f$ and $p d f$ of TIIHL-G are given, respectively, by

$$
F(x ; \lambda, \zeta)=\frac{2[G(x ; \zeta)]^{\lambda}}{1+[G(x ; \zeta)]^{\lambda}}, \quad x \geq 0,
$$

and

$$
f(x ; \lambda, \zeta)=\frac{2 \lambda g(x ; \zeta)[G(x ; \zeta)]^{\lambda-1}}{\left[1+[G(x ; \zeta)]^{\lambda}\right]^{2}}, \quad x>0,
$$

where $\lambda$ is a positive parameter and $G(x ; \zeta), g(x ; \zeta)$ are $c d f$ and $p d f$ of the baseline distribution which depends on the parameter vector $\zeta$.

11) The $c d f$ and $p d f$ of TNGIW are given, respectively, by

$$
F(x ; \alpha, \beta, \gamma, \phi, \lambda)=\left\{1-\left[1-\exp \left\{-\frac{\alpha}{x}-\gamma\left(\frac{1}{x}\right)^{\beta}\right\}\right]^{\phi}\right\}\left\{1+\lambda\left[1-\exp \left\{-\frac{\alpha}{x}-\gamma\left(\frac{1}{x}\right)^{\beta}\right\}\right]^{\phi}\right\}
$$

$x \geq 0$ and

$$
\begin{gathered}
f(x ; \alpha, \beta, \gamma, \phi, \lambda)=\phi\left\{\frac{\alpha}{x^{2}}+\beta \gamma\left(\frac{1}{x}\right)^{\beta+1}\right\} \exp \left\{-\frac{\alpha}{x}-\gamma\left(\frac{1}{x}\right)^{\beta}\right\} \times \\
{\left[1-\exp \left\{-\frac{\alpha}{x}-\gamma\left(\frac{1}{x}\right)^{\beta}\right\}\right]^{\phi-1} \times} \\
\left\{1-\lambda+2 \lambda\left[1-\exp \left\{-\frac{\alpha}{x}-\gamma\left(\frac{1}{x}\right)^{\beta}\right\}\right]^{\phi}\right\},
\end{gathered}
$$

$x>0$, where $\alpha, \beta, \gamma, \phi$ all positive and $\lambda(|\lambda| \leq 1)$ are parameters.

Remark 4. For $\lambda=0$, TNGIW will reduce to NGIW (New Generalized Inverse Weibull) distribution of Khan and King (2016).

12) The $c d f$ and $p d f$ of Po-G are given, respectively, by

$$
F(x ; \theta, \eta)=\frac{\exp [\theta G(x ; \eta)]-1}{e^{\theta}-1}, \quad x \in \mathbb{R},
$$

and

$$
f(x ; \theta, \eta)=\frac{\theta g(x ; \eta) \exp [\theta G(x ; \eta)]}{e^{\theta}-1}, \quad x \in \mathbb{R},
$$

where $\theta$ is a positive parameter and $g(x ; \eta)$ and $G(x ; \eta)$ are $p d f$ and $c d f$ of the baseline distribution which depends on the parameter vector $\eta$. 
13) The $c d f$ and $p d f$ of GTW are given, respectively, by

$$
F(x ; \alpha, \beta, a, b, \lambda)=\left[1-e^{-(\alpha x)^{\beta}}\right]\left\{1+\lambda-\lambda\left[1-e^{-(\alpha x)^{\beta}}\right]^{b}\right\}, x \geq 0,
$$

and

$$
f(x ; \alpha, \beta, a, b, \lambda)=\beta \alpha^{\beta} x^{\beta-1} e^{-(\alpha x)^{\beta}}\left[1-e^{-(\alpha x)^{\beta}}\right]^{a-1}\left\{a(1+\lambda)-\lambda(a+b)\left[1-e^{-(\alpha x)^{\beta}}\right]^{b}\right\},
$$

$x>0$, where $\alpha, \beta, a, b$ all positive and $\lambda(|\lambda| \leq 1)$ are parameters.

14) The $c d f$ and $p d f$ of EGGP are given, respectively, by

$$
F(x ; a, b, \lambda, \psi)=\frac{1-\exp \left\{-\lambda\left\{1-[\bar{G}(x ; \psi)]^{a}\right\}^{b}\right\}}{1-\exp \{-\lambda\}}, \quad x \in \mathbb{R},
$$

and

$$
f(x ; a, b, \lambda, \psi)=\frac{a b \lambda g(x ; \psi)[\bar{G}(x ; \psi)]^{a-1}\left\{1-[\bar{G}(x ; \psi)]^{a}\right\}^{b-1}}{\{1-\exp \{-\lambda\}\} \exp \left\{\lambda\left\{1-[\bar{G}(x ; \psi)]^{a}\right\}^{b}\right\}},
$$

$x \in \mathbb{R}$, where $a, b, \lambda$ are positive parameters and $g(x ; \psi)$ and $G(x ; \psi)$ are $p d f$ and $c d f$ of the baseline distribution which depends on the parameter vector $\psi$.

15) The $c d f$ and $p d f$ of BIR are given, respectively, by

$$
F(x ; a, b, \theta)=\frac{1}{B(a, b)} \int_{0}^{\exp \left(-\theta x^{-2}\right)} w^{a-1}(1-w)^{b-1} d w, x \geq 0,
$$

and

$$
f(x ; a, b, \theta)=\frac{2 \theta}{B(a, b)} x^{-3} \exp \left(-\theta x^{-2}\right)\left[1-\exp \left(-\theta x^{-2}\right)\right]^{b-1}, x>0,
$$

where $a, b, \theta$ are positive parameters.

16) The $c d f$ and $p d f$ of $\operatorname{BIR}_{1}\left(a=b=\frac{1}{2}\right)$ are given, respectively, by

$$
F(x ; \theta)=\frac{2}{\pi} \arcsin \left\{\exp \left(-\frac{\theta}{2} x^{-2}\right)\right\}, \quad x \geq 0
$$

and

$$
f(x ; \theta)=\frac{2}{\pi}\left\{\frac{\theta x^{-3} \exp \left(-\frac{\theta}{2} x^{-2}\right)}{\sqrt{1-\exp \left(-\theta x^{-2}\right)}}\right\}, \quad x>0,
$$

where $\theta$ is a positive parameter.

17) The $c d f$ and $p d f$ of BWP are given, respectively, by

$$
F(x ; \alpha, \beta, a, b, \lambda)=\frac{1}{B(a, b)} \int_{0}^{\frac{e^{\lambda}-\beta x^{\alpha}}{1-e^{l}}-e^{\lambda}} w^{a-1}(1-w)^{b-1} d w, x \geq 0,
$$

and

$$
f(x ; \theta)=c x^{\alpha-1} e^{-\beta x^{\alpha}} e^{\lambda e^{-\beta x^{\alpha}}}\left(e^{\lambda e^{-\beta x^{\alpha}}}-1\right)^{b-1}, \quad x>0,
$$

where $\alpha, \beta, a, b, \lambda$ are positive parameters and $c=\frac{\alpha \beta \lambda e^{-\lambda}\left(e^{\lambda}-1\right)^{2-a-b}}{B(a, b)\left(1-e^{-\lambda}\right)}$ is the normalizing constant. Note that for $a=1$, $c=b \alpha \beta \lambda\left(e^{\lambda}-1\right)^{-b}$ and for $b=1, c=a \alpha \beta \lambda\left(e^{\lambda}-1\right)^{-a}$.

18) The $c d f$ and $p d f$ of GMW are given, respectively, by

$$
F(x ; \alpha, \beta, a, \lambda)=\frac{1}{\Gamma(a)} \int_{0}^{\alpha x^{\beta} e^{\lambda x}} t^{a-1} e^{-t} d t, \quad x \geq 0,
$$


and

$$
f(x ; \alpha, \beta, a, \lambda)=\frac{\alpha x^{\beta-1}(\beta+\lambda x)}{\Gamma(a)}\left\{\alpha x^{\beta} e^{\lambda x}\right\}^{a-1} e^{\lambda x-\alpha x^{\beta} e^{\lambda x}}, x>0,
$$

where $\alpha, \beta, a, \lambda$ are positive parameters.

19) The $c d f$ and $p d f$ of TD are given, respectively, by

$$
F(x ; \alpha, \beta, \theta, \lambda)=\left(1+\alpha x^{-\theta}\right)^{-\beta}\left[1+\lambda-\lambda\left(1+\alpha x^{-\theta}\right)^{-\beta}\right], \quad x \geq 0,
$$

and

$$
f(x ; \alpha, \beta, \theta, \lambda)=\alpha \beta \theta x^{-\theta-1}\left(1+\alpha x^{-\theta}\right)^{-\beta-1}\left[1+\lambda-2 \lambda\left(1+\alpha x^{-\theta}\right)^{-\beta}\right],
$$

$x>0$, where $\alpha, \beta, \theta$ positive and $\lambda(|\lambda| \leq 1)$ are parameters.

20) The $c d f$ and $p d f$ of EGG are given, respectively, by

$$
F(x ; \alpha, \theta, \gamma, \eta)=\left\{1-e^{\frac{\theta}{\gamma}\left(1-(\bar{G}(x ; \eta))^{-\gamma}\right)}\right\}^{\alpha}, \quad x \in \mathbb{R}
$$

and

$$
f(x ; \alpha, \theta, \gamma, \eta)=\frac{\alpha \theta g(x ; \eta) e^{\frac{\theta}{\gamma}\left(1-(\bar{G}(x ; \eta))^{-\gamma}\right)}}{(\bar{G}(x ; \eta))^{1+\gamma}\left\{1-e^{\frac{\theta}{\gamma}\left(1-(\bar{G}(x ; \eta))^{-\gamma}\right)}\right\}^{1-\alpha}}, \quad x \in \mathbb{R},
$$

where $\alpha, \theta, \gamma$ are positive parameters and $g(x ; \eta), G(x ; \eta)$ are $p d f$ and $c d f$ of the baseline distribution with parameter vector $\eta$.

21) The $c d f$ and $p d f$ of NEPL are given, respectively, by

$$
F(x ; \alpha, \beta, \lambda)=\frac{\left[1-\left(1+\frac{\lambda}{1+\lambda} x^{\beta}\right) e^{-\lambda x^{\beta}}\right]^{\alpha}}{\left[1-\left(1+\frac{\lambda}{1+\lambda} x^{\beta}\right) e^{-\lambda x^{\beta}}\right]^{\alpha}+\left(1+\frac{\lambda}{1+\lambda} x^{\beta}\right) e^{-\lambda x^{\beta}}}, x \geq 0,
$$

and

$$
f(x ; \alpha, \beta, \lambda)=\frac{\alpha \beta \lambda^{2}\left(1+x^{\beta}\right) e^{-\lambda x^{\beta}}\left[1-\left(1+\frac{\lambda}{1+\lambda} x^{\beta}\right) e^{-\lambda x^{\beta}}\right]^{\alpha-1}\left(1+\frac{\lambda}{1+\lambda} x^{\beta}\right)^{\alpha-1}}{(1+\lambda)\left\{\left[1-\left(1+\frac{\lambda}{1+\lambda} x^{\beta}\right) e^{-\lambda x^{\beta}}\right]^{\alpha}+\left(1+\frac{\lambda}{1+\lambda} x^{\beta}\right) e^{-\lambda x^{\beta}}\right\}^{2}}, x>0
$$

where $\alpha, \beta, \lambda$ are positive parameters.

22) The $c d f$ and $p d f$ of PWR (WLOG for $\mu=0, \sigma=1$ ) are given, respectively, by

$$
F(x ; \alpha)=\frac{e^{\alpha}-e^{\alpha e^{-e^{x}}}}{e^{\alpha}-1}, \quad x \in \mathbb{R},
$$

and

$$
f(x ; \alpha)=\frac{\alpha}{e^{\alpha}-1} e^{x+\alpha e^{-e^{x}}-e^{x}}, \quad x \in \mathbb{R},
$$

where $\alpha$ is a positive parameter.

23) The $c d f$ and $p d f$ of OLLSN (WLOG for $\mu=0, \sigma=1$ ) are given, respectively, by

$$
F(x ; \lambda)=\frac{\left[\Phi_{S N}(x ; \lambda)\right]^{\alpha}}{\left[\Phi_{S N}(x ; \lambda)\right]^{\alpha}+\left[\bar{\Phi}_{S N}(x ; \lambda)\right]^{\alpha}}, \quad x \in \mathbb{R},
$$

and

$$
f(x ; \lambda)=\frac{2 \alpha \phi(x) \Phi(\lambda x)\left[\Phi_{S N}(x ; \lambda)\right]^{\alpha-1}\left[\bar{\Phi}_{S N}(x ; \lambda)\right]^{\alpha-1}}{\left\{\left[\Phi_{S N}(x ; \lambda)\right]^{\alpha}+\left[\bar{\Phi}_{S N}(x ; \lambda)\right]^{\alpha}\right\}^{2}}, \quad x \in \mathbb{R}
$$


where $\lambda$ is a positive parameter, $\phi(x), \Phi(x)$ are $p d f$ and $c d f$ of the standard normal distribution, $\Phi_{S N}(x ; \lambda)=\Phi(x)-$ $2 T(x ; \lambda), T(x ; \lambda)=(2 \pi)^{-1} \int_{0}^{\lambda} \frac{\exp \left\{-\frac{1}{2} x^{2}\left(1+u^{2}\right)\right\}}{1+u^{2}} d u$ and $\bar{\Phi}_{S N}(x ; \lambda)=1-\Phi_{S N}(x ; \lambda)$.

24) The $c d f$ and $p d f$ of EGGG are given, respectively, by

$$
F(x ; \alpha, \tau, \lambda, p, k)=1-\left\{1-\frac{\gamma_{1}\left[k,\left(\frac{x}{\alpha}\right)^{\tau}\right]}{1-p\left\{1-\gamma_{1}\left[k,\left(\frac{x}{\alpha}\right)^{\tau}\right]\right\}}\right\}^{\lambda}, \quad x \geq 0,
$$

and

$$
f(x ; \alpha, \tau, \lambda, p, k)=\frac{\lambda \tau(1-p)^{\lambda}}{\alpha \Gamma(k)}\left(\frac{x}{\alpha}\right)^{\tau k-1} \exp \left[-\left(\frac{x}{\alpha}\right)^{\tau}\right] \frac{\left\{\left(1-\gamma_{1}\left[k,\left(\frac{x}{\alpha}\right)^{\tau}\right]\right)\left(1-p\left\{1-\gamma_{1}\left[k,\left(\frac{x}{\alpha}\right)^{\tau}\right]\right\}\right)\right\}^{\lambda-1}}{\left(1-p\left\{1-\gamma_{1}\left[k,\left(\frac{x}{\alpha}\right)^{\tau}\right]\right\}\right)^{2}},
$$

$x>0$, where $\alpha, \tau, \lambda, k$ positive, $p \in(0,1)$ are parameters, $\gamma[k, x]=\int_{0}^{x} w^{k-1} e^{-w} d w, \gamma_{1}[k, x]=\frac{1}{\Gamma(k)} \int_{0}^{x} w^{k-1} e^{-w} d w$ and $\Gamma(k)=\int_{0}^{\infty} w^{k-1} e^{-w} d w$.

25) The $c d f$ and $p d f$ of ETQL are given, respectively, by

$$
F(x ; \alpha, \beta, \mu, \theta, \lambda)=\left[1-\left\{1-\left[\begin{array}{c}
(1+\lambda)\left(1-\frac{\mu \theta+1+\theta x}{\mu \theta+1} e^{-\theta x}\right) \\
-\lambda\left(1-\frac{\mu \theta+1+\theta x}{\mu \theta+1} e^{-\theta x}\right)^{2}
\end{array}\right]\right\}^{\alpha}\right]^{\beta}, \quad x \geq 0,
$$

and

$$
\begin{aligned}
f(x ; \alpha, \beta, \mu, \theta, \lambda)=\frac{\alpha \beta \theta^{2}}{\mu \theta+1} e^{-\theta x}(\mu+x)\{1 & \left.-\left[\begin{array}{c}
(1+\lambda)\left(1-\frac{\mu \theta+1+\theta x}{\mu \theta+1} e^{-\theta x}\right) \\
-\lambda\left(1-\frac{\mu \theta+1+\theta x}{\mu \theta+1} e^{-\theta x}\right)^{2}
\end{array}\right]\right\}^{\alpha-1}\left[1-\left\{1-\left[\begin{array}{c}
(1+\lambda)\left(1-\frac{\mu \theta+1+\theta x}{\mu \theta+1} e^{-\theta x}\right) \\
-\lambda\left(1-\frac{\mu \theta+1+\theta x}{\mu \theta+1} e^{-\theta x}\right)^{2}
\end{array}\right]\right\}^{\alpha}\right]^{\beta-1} \\
& \times\left[1+\lambda-2 \lambda\left(1-\frac{\mu \theta+1+\theta x}{\mu \theta+1} e^{-\theta x}\right)\right]
\end{aligned}
$$

$x>0$, where $\alpha, \beta, \mu, \theta$ positive and $\lambda(|\lambda| \leq 1)$ are parameters.

26) The $p d f$ of LKGG (WLOG for $\mu=0, \sigma=1$ ) is given, respectively, by

$$
f(x ; \lambda, \varphi, q)=\left\{\begin{array}{c}
\frac{\lambda q q q \mid}{\Gamma\left(q^{-2}\right)}\left(q^{-2}\right)^{q^{-2}} \exp \left\{q^{-1} x-q^{-2} e^{q x}\right\} \times \\
\left\{\gamma_{1}\left[q^{-2}, q^{-2} e^{q x}\right]\right\}^{\lambda-1}\left\{1-\left(\gamma_{1}\left[q^{-2}, q^{-2} e^{q x}\right]\right)^{\lambda}\right\}^{\varphi-1}, \quad \text { if } q \neq 0 \\
\frac{\lambda \varphi}{\sqrt{2 \pi}} \exp \left(-\frac{x^{2}}{2}\right)(\Phi(x))^{\lambda-1}\left[1-(\Phi(x))^{\lambda}\right]^{\varphi-1}, \quad \text { if } q=0
\end{array}\right.
$$

$x \in \mathbb{R}$, where $\lambda, \varphi$ positive,$q \in \mathbb{R}$ are parameters and, as before, $\gamma_{1}[\alpha, x]=\frac{1}{\Gamma(\alpha)} \int_{0}^{x} w^{\alpha-1} e^{-w} d w$.

27) The $c d f$ and $p d f$ of ERK are given, respectively, by

$$
F(x ; \beta, \lambda)=\left\{1-\exp \left[-\left(\frac{x}{1-x}\right)^{\beta}\right]\right\}^{\lambda}, \quad 0 \leq x \leq 1,
$$

and

$$
f(x ; \beta, \lambda)=\frac{\lambda \beta x^{\beta-1} \exp \left[-\left(\frac{x}{1-x}\right)^{\beta}\right]}{(1-x)^{\beta+1}}\left\{1-\exp \left[-\left(\frac{x}{1-x}\right)^{\beta}\right]\right\}^{\lambda-1},
$$

$0<x<1$, where $\beta, \lambda$ are positive parameters.

28) The $c d f$ and $p d f$ of EL are given, respectively, by

$$
F(x ; \alpha, \theta, \lambda)=\left[1-(1+\lambda x)^{-\theta}\right]^{\alpha}, \quad x \geq 0,
$$


and

$$
f(x ; \alpha, \theta, \lambda)=\alpha \theta \lambda(1+\lambda x)^{-(\theta+1)}\left[1-(1+\lambda x)^{-\theta}\right]^{\alpha-1}, \quad x>0,
$$

where $\alpha, \theta, \lambda$ are positive parameters.

Remark 5. A generalization of the EL distribution was proposed by Mead (2015) which is characterized in the upcoming Research Monograph by Hamedani and Maadooliat (2017).

29) The $c d f$ and $p d f$ of AEE are given, respectively, by

$$
F(x ; \alpha, \beta)=1-\frac{(\alpha+\beta+\alpha \beta x) e^{-\alpha x}}{\alpha+\beta}, \quad x \geq 0,
$$

and

$$
f(x ; \alpha, \beta)=\frac{\alpha^{2}(1+\beta x) e^{-\alpha x}}{\alpha+\beta}, \quad x>0,
$$

where $\alpha, \beta$ are positive parameters.

30) The $c d f$ and $p d f$ of NWE are given, respectively, by

$$
F(x ; \alpha, \beta, \lambda)=1-\frac{C}{\lambda} e^{-\lambda x}\left\{\frac{\beta}{\beta+1}-\frac{e^{-\lambda \alpha x}}{\alpha+1}+\frac{e^{-\lambda \alpha(\beta+1) x}}{(\beta+1)(1+\alpha(\beta+1))}\right\}, \quad x \geq 0
$$

and

$$
f(x ; \alpha, \beta, \lambda)=C e^{-\lambda x}\left\{1-e^{-\lambda \alpha x}-\frac{\left(1-e^{-\lambda \alpha(\beta+1) x}\right)}{(\beta+1)}\right\}, \quad x>0,
$$

where $\alpha, \beta, \lambda$ are positive parameters and $C=\frac{\lambda(\alpha+1)(1+\alpha(\beta+1))}{\beta \alpha^{2}}$ is the normalizing constant.

31) The $c d f$ and $p d f$ of EIWG are given, respectively, by

$$
F(x ; \alpha, \gamma, q)=\frac{e^{-\gamma x^{-\alpha}}}{1-q\left(1-e^{-\gamma x^{-\alpha}}\right)}, \quad x \geq 0,
$$

and

$$
f(x ; \alpha, \gamma, q)=\frac{\alpha \gamma(1-q) x^{-(\alpha+1)} e^{-\gamma x^{-\alpha}}}{\left[1-q\left(1-e^{-\gamma x^{-\alpha}}\right)\right]^{2}}, \quad x>0,
$$

where $\alpha, \gamma$ positive and $q(0<q<1)$ are parameters.

Remark 6. The EIWG distribution is a special case of BGIWG (The Beta Generalized Inverse Weibull Geometric) distribution of Elbatal et al. (2017). We believe that Chung et al. were not aware of Elbatal et al.'s paper as these two papers were published within a couple of months of each other. The BGIWG distribution was characterized in Hamedani (2017) paper listed in the references here.

32) The $c d f$ and $p d f$ of NEE are given, respectively, by

$$
F(x ; \alpha, \beta)=1-\frac{\alpha^{2}(1+\beta x) e^{-\alpha x}}{\alpha+\beta}, \quad x \geq 0,
$$

and

$$
f(x ; \alpha, \beta)=\frac{(\alpha+\beta+\alpha \beta x) e^{-\alpha x}}{\alpha+\beta}, \quad x>0,
$$

where $\alpha, \beta$ are positive parameters.

33) The $c d f$ and $p d f$ of ETW are given, respectively, by

$$
F(x ; \alpha, \beta, \lambda, v)=\left\{1+(\lambda-1) e^{-\left(\frac{x}{\alpha}\right)^{\beta}}-\lambda e^{-2\left(\frac{x}{\alpha}\right)^{\beta}}\right\}^{v}, x \geq 0,
$$

and

$$
f(x ; \alpha, \beta, \lambda, v)=\frac{v \beta}{\alpha}\left(\frac{x}{\alpha}\right)^{\beta-1} e^{-\left(\frac{x}{\alpha}\right)^{\beta}}\left[1-\lambda+2 \lambda e^{-\left(\frac{x}{\alpha}\right)^{\beta}}\right]\left\{1+(\lambda-1) e^{-\left(\frac{x}{\alpha}\right)^{\beta}}-\lambda e^{-2\left(\frac{x}{\alpha}\right)^{\beta}}\right\}^{\nu-1}
$$


$x>0$, where $\alpha, \beta, v$ all positive and $\lambda(|\lambda| \leq 1)$ are parameters.

34) The $c d f$ and $p d f$ of GW are given, respectively, by

$$
F(x ; \alpha, \beta)=\frac{1-e^{-x^{\beta}}}{1-(1-\alpha) e^{-x^{\beta}}}, \quad x \geq 0,
$$

and

$$
f(x ; \alpha, \beta)=\frac{\alpha \beta x^{\beta-1} e^{-x^{\beta}}}{\left[1-(1-\alpha) e^{-x^{\beta}}\right]^{2}}, \quad x>0,
$$

where $\alpha, \beta, v$ are positive parameters.

Remark 7. Generalizations of GW distributions were presented by Bidram et al. (2015) and by Elbatal et al. (2016). These distributions are characterized in the upcoming Research Monograph by Hamedani and Maadooliat (2017).

35) The $c d f$ and $p d f$ of ENHE are given, respectively, by

$$
F(x ; \alpha, \beta, \lambda)=\left(1-e^{1-(1+\alpha x)^{\beta}}\right)^{\lambda}, \quad x \geq 0,
$$

and

$$
f(x ; \alpha, \beta, \lambda)=\lambda \alpha \beta(1+\alpha x)^{\beta-1} e^{1-(1+\alpha x)^{\beta}}\left(1-e^{1-(1+\alpha x)^{\beta}}\right)^{\lambda-1},
$$

$x>0$, where $\alpha, \beta, \lambda$ are all positive parameters.

36) The $c d f$ and $p d f$ of GW are given, respectively, by

$$
F(x ; \alpha, \theta, c)=\frac{1}{1-\alpha^{\theta}}\left\{1-\alpha^{\theta}\left(1-e^{-x^{c}}+\alpha e^{-x^{c}}\right)^{-\theta}\right\}, x \geq 0,
$$

and

$$
f(x ; \alpha, \theta, c)=\frac{\theta(1-\alpha) \alpha^{\theta} c x^{c-1} e^{-x^{c}}}{\left(1-\alpha^{\theta}\right)\left(1-e^{-x^{c}}+\alpha e^{-x^{c}}\right)^{\theta+1}}, x>0,
$$

where $\alpha, \theta, c$ are all positive parameters.

37) The $c d f$ and $p d f$ of TW-G are given, respectively, by

$$
F(x ; \alpha, \beta)=\frac{1-\exp \left[-\alpha G(x)^{\beta}\right]}{1-\exp (-\alpha)}, \quad x \in \mathbb{R},
$$

and

$$
f(x ; \alpha, \beta)=\frac{\alpha \beta g(x) G(x)^{\beta-1} \exp \left[-\alpha G(x)^{\beta}\right]}{1-\exp (-\alpha)}, \quad x \in \mathbb{R},
$$

where $\alpha, \beta$ are positive parameters.

Remark 8. The TW-G distribution is not new. It was first introduced by Gomes et al. (2015), which is also characterized in the upcoming Research Monograph by Hamedani and Maadooliat (2017).

38) The $c d f$ and $p d f$ of GAW-G are given, respectively, by

$$
F(x ; a, b, c, d, \eta)=1-\exp \left\{-c[-\ln (\bar{G}(x ; \eta))]^{d}-a[-\ln (\bar{G}(x ; \eta))]^{b}\right\}, x \in \mathbb{R},
$$

and

$f(x ; a, b, c, d, \eta)=\frac{g(x ; \eta)}{\bar{G}(x ; \eta)}\left\{c d[-\ln (\bar{G}(x ; \eta))]^{d-1}+a b[-\ln (\bar{G}(x ; \eta))]^{b-1}\right\} \exp \left\{-c[-\ln (\bar{G}(x ; \eta))]^{d}-a[-\ln (\bar{G}(x ; \eta))]^{b}\right\}$,

$x \in \mathbb{R}$, where $a, b, c, d$ are positive parameters, $G(x ; \eta)$ and $g(x ; \eta)$ are $c d f$ and $p d f$ of the baseline distribution which depends on the parameter vector $\eta$. 
39) The $c d f$ and $p d f$ of TWE are given, respectively, by

$$
F\left(x ; \lambda, \alpha_{1}, \alpha_{2}\right)=1-C e^{-\lambda x}\left(\begin{array}{c}
1-\frac{1}{1+\alpha_{1}} e^{-\lambda \alpha_{1} x}-\frac{1}{1+\alpha_{2}} e^{-\lambda \alpha_{2} x} \\
+\frac{1}{1+\alpha_{1}+\alpha_{2}} e^{-\lambda\left(\alpha_{1}+\alpha_{2}\right) x}
\end{array}\right), \quad x \geq 0,
$$

and

$$
f\left(x ; \lambda, \alpha_{1}, \alpha_{2}\right)=C \lambda e^{-\lambda x}\left(1-e^{-\lambda \alpha_{1} x}\right)\left(1-e^{-\lambda \alpha_{2} x}\right), \quad x>0,
$$

where $\lambda, \alpha_{1}, \alpha_{2}$ are positive parameters and $C=\frac{\left(1+\alpha_{1}\right)\left(1+\alpha_{2}\right)\left(1+\alpha_{1}+\alpha_{2}\right)}{\alpha_{1} \alpha\left(2+\alpha_{1}+\alpha_{2}\right)}$ is the normalizing constant.

40) The $p d f$ of GWW is given by

$$
f(x ; \alpha, \beta, \lambda, v)=C \lambda \beta x^{\beta-1} e^{-\lambda x^{\beta}}\left(1-e^{-\lambda(\alpha x)^{\beta}}\right)^{v}, \quad x>0,
$$

where $\alpha, \beta, \lambda, v$ are positive parameters and $C=\frac{\alpha^{\beta}}{B\left(\frac{1}{a^{\beta}}, v+1\right)}$ is the normalizing constant and $B(a, b)$ is the beta function.

Remark 9. The Generalized Weighted Exponential (GWE) distribution of Kharazmi et al. (2017) is a special case of GWW distribution.

41) The $c d f$ and $p d f$ of GB are given, respectively, by

$$
F(x ; \theta, \lambda)=1-e^{-2(x / \theta)^{\lambda}}\left(3-2 e^{-(x / \theta)^{\lambda}}\right), \quad x \geq 0,
$$

and

$$
f(x ; \theta, \lambda)=\frac{6 \lambda}{\theta}\left(\frac{x}{\theta}\right)^{\lambda-1} e^{-2(x / \theta)^{\lambda}}\left(1-e^{-(x / \theta)^{\lambda}}\right), \quad x>0,
$$

where $\theta, \lambda$ are positive parameters.

\section{Characterizations of Distributions}

We present our characterizations (i) - (iv) in four subsections.

\subsection{Characterizations Based on Two Truncated Moments}

This subsection deals with the characterizations of distributions listed in Section 1 based on the ratio of two truncated moments. Our first characterization employs a theorem due to Glänzel (1987), see Theorem 1 of Appendix A . The result, however, holds also when the interval $H$ is not closed, since the condition of the Theorem is on the interior of $H$.

Remark 1.1. For $\beta=0$, the distribution (1) has the simple form which has been characterized in our previous work. We will concentrate on the following three cases: $I) \beta<0$;II) $\beta>0$ and $b \neq 0$ and III) $\beta>0$ and $b=0$.

CASE $I$ :

Proposition 1.1. Let $X: \Omega \rightarrow(0, \infty)$ be a continuous random variable and let $q_{2}(x)=\left(1-\left(1-\beta\left(a x+\frac{b}{2} x^{2}\right)\right)^{1 / \beta}\right)^{1-\alpha}$ and $q_{1}(x)=q_{2}(x)\left(1-\beta\left(a x+\frac{b}{2} x^{2}\right)\right)^{-1}$ for $x>0$. Then for $\beta<0$, the random variable $X$ has $p d f(2)$ if and only if the function $\xi$ defined in Theorem 1 is of the form

$$
\xi(x)=(1-\beta)\left(1-\beta\left(a x+\frac{b}{2} x^{2}\right)\right), \quad x>0 .
$$

Proof. Suppose the random variable $X$ has $p d f(2)$, then

$$
(1-F(x)) E\left[q_{1}(X) \mid X \geq x\right]=\frac{\alpha}{1-\beta}\left(1-\beta\left(a x+\frac{b}{2} x^{2}\right)\right)^{\frac{1}{\beta}-1}, \quad x>0,
$$

and

$$
(1-F(x)) E\left[q_{2}(X) \mid X \geq x\right]=\alpha\left(1-\beta\left(a x+\frac{b}{2} x^{2}\right)\right)^{1 / \beta}, \quad x>0 .
$$


Further,

$$
\xi(x) q_{1}(x)-q_{2}(x)=-\beta q_{2}(x)>0, \text { for } x>0
$$

Conversely, if $\xi$ is of the above form, then

$$
s^{\prime}(x)=\frac{\xi^{\prime}(x) q_{1}(x)}{\xi(x) q_{1}(x)-q_{2}(x)}=\frac{(1-\beta)(a+b x)}{1-\beta\left(a x+\frac{b}{2} x^{2}\right)}, \quad x>0,
$$

and consequently

$$
s(x)=-\frac{1-\beta}{\beta} \log \left\{1-\beta\left(a x+\frac{b}{2} x^{2}\right)\right\}, \quad x>0 .
$$

Now, according to Theorem 1, $X$ has density (2).

Corollary 1.1. Let $X: \Omega \rightarrow(0, \infty)$ be a continuous random variable and let $q_{2}(x)$ be as in Proposition 1.1 . For $\beta<0$, the random variable $X$ has $p d f(2)$ if and only if there exist functions $q_{1}$ and $\xi$ defined in Theorem 1 satisfying the following differential equation

$$
\frac{\xi^{\prime}(x) q_{1}(x)}{\xi(x) q_{1}(x)-q_{2}(x)}=\frac{(1-\beta)(a+b x)}{1-\beta\left(a x+\frac{b}{2} x^{2}\right)}, \quad x>0
$$

Remark 1.2. The general solution of the differential equation in Corollary 1.1 is

$$
\xi(x)=\left[1-\beta\left(a x+\frac{b}{2} x^{2}\right)\right]^{1-\frac{1}{\beta}}\left[\begin{array}{c}
-\int(1-\beta)(a+b x)\left[1-\beta\left(a x+\frac{b}{2} x^{2}\right)\right]^{\frac{1}{\beta}-2} \times \\
\left(q_{1}(x)\right)^{-1} q_{2}(x) d x+D
\end{array}\right]
$$

where $D$ is a constant. We like to point out that one set of functions satisfying the above differential equation is given in Proposition 1.1 with $D=0$. Clearly, there are other triplets $\left(q_{1}, q_{2}, \xi\right)$ which satisfy conditions of Theorem 1 .

\section{CASE II :}

Proposition 1.2. Let $X: \Omega \rightarrow\left(0, \frac{1}{b} \sqrt{a^{2}+\frac{2 b}{\beta}}-\frac{a}{b}\right)$ be a continuous random variable and let $q_{1}(x)$ and $q_{2}(x)$ be as in Proposition 1.1 for $0<x<\frac{1}{b} \sqrt{a^{2}+\frac{2 b}{\beta}}-\frac{a}{b}$. Then for $\beta>0$ and $b \neq 0$, the random variable $X$ has $p d f$ (2) if and only if the function $\xi$ defined in Theorem 1 is of the form

$$
\xi(x)=(1-\beta)\left(1-\beta\left(a x+\frac{b}{2} x^{2}\right)\right), \quad 0<x<\frac{1}{b} \sqrt{a^{2}+\frac{2 b}{\beta}}-\frac{a}{b} .
$$


Proof. Suppose the random variable $X$ has $p d f$ (2), then

$$
\begin{aligned}
(1-F(x)) E\left[q_{2}(X) \mid X \geq x\right] & =\int_{x}^{\frac{1}{b} \sqrt{a^{2}+\frac{2 b}{\beta}}-\frac{a}{b}} \alpha(a+b u)\left(1-\beta\left(a u+\frac{b}{2} u^{2}\right)\right)^{\frac{1}{\beta}-1} d u \\
& =-\left.\alpha\left(1-\beta\left(a u+\frac{b}{2} u^{2}\right)\right)^{1 / \beta}\right|_{x} ^{\frac{1}{b} \sqrt{a^{2}+\frac{2 b}{\beta}}-\frac{a}{b}} \\
& =-\alpha\left(1-\beta\left(\begin{array}{c}
a\left(\frac{1}{b} \sqrt{a^{2}+\frac{2 b}{\beta}}-\frac{a}{b}\right)+ \\
\frac{b}{2}\left(\frac{1}{b} \sqrt{a^{2}+\frac{2 b}{\beta}}-\frac{a}{b}\right)^{2}
\end{array}\right)\right)^{1 / \beta}+ \\
& \alpha\left(1-\beta\left(a x+\frac{b}{2} x^{2}\right)\right)^{1 / \beta} \\
& =-\alpha\left(1-\beta\left(\frac{1}{\beta}\right)\right)^{1 / \beta}+\alpha\left(1-\beta\left(a x+\frac{b}{2} x^{2}\right)\right)^{1 / \beta} \\
& =\alpha\left(1-\beta\left(a x+\frac{b}{2} x^{2}\right)\right)^{1 / \beta}, \quad 0<x<\frac{1}{b} \sqrt{a^{2}+\frac{2 b}{\beta}}-\frac{a}{b} .
\end{aligned}
$$

Similarly,

$$
(1-F(x)) E\left[q_{1}(X) \mid X \geq x\right]=\frac{\alpha}{1-\beta}\left(1-\beta\left(a x+\frac{b}{2} x^{2}\right)\right)^{\frac{1}{\beta}-1}, 0<x<\frac{1}{b} \sqrt{a^{2}+\frac{2 b}{\beta}}-\frac{a}{b} .
$$

Further,

$$
\xi(x) q_{1}(x)-q_{2}(x)=-\beta q_{2}(x)<0, \text { for } 0<x<\frac{1}{b} \sqrt{a^{2}+\frac{2 b}{\beta}}-\frac{a}{b} .
$$

Conversely, if $\xi$ is of the above form, then

$$
s^{\prime}(x)=\frac{\xi^{\prime}(x) q_{1}(x)}{\xi(x) q_{1}(x)-q_{2}(x)}=\frac{(1-\beta)(a+b x)}{1-\beta\left(a x+\frac{b}{2} x^{2}\right)}, \quad 0<x<\frac{1}{b} \sqrt{a^{2}+\frac{2 b}{\beta}}-\frac{a}{b},
$$

and consequently

$$
s(x)=-\frac{1-\beta}{\beta} \log \left\{1-\beta\left(a x+\frac{b}{2} x^{2}\right)\right\}, \quad 0<x<\frac{1}{b} \sqrt{a^{2}+\frac{2 b}{\beta}}-\frac{a}{b} .
$$

Now, according to Theorem 1, $X$ has density (2).

Corollary 1.2. Let $X: \Omega \rightarrow\left(0, \frac{1}{b} \sqrt{a^{2}+\frac{2 b}{\beta}}-\frac{a}{b}\right)$ be a continuous random variable and let $q_{2}(x)$ be as in Proposition 1.2. For $\beta>0$ and $b \neq 0$, the random variable $X$ has $p d f(2)$ if and only if there exist functions $q_{1}$ and $\xi$ defined in Theorem 1 satisfying the following differential equation

$$
\frac{\xi^{\prime}(x) q_{1}(x)}{\xi(x) q_{1}(x)-q_{2}(x)}=\frac{(1-\beta)(a+b x)}{1-\beta\left(a x+\frac{b}{2} x^{2}\right)}, \quad 0<x<\frac{1}{b} \sqrt{a^{2}+\frac{2 b}{\beta}}-\frac{a}{b} .
$$

Remark 1.3. The general solution of the differential equation in Corollary 1.2 is

$$
\xi(x)=\left[1-\beta\left(a x+\frac{b}{2} x^{2}\right)\right]^{1-\frac{1}{\beta}}\left[\begin{array}{c}
-\int(1-\beta)(a+b x)\left[1-\beta\left(a x+\frac{b}{2} x^{2}\right)\right]^{\frac{1}{\beta}-2} \times \\
\left(q_{1}(x)\right)^{-1} q_{2}(x) d x+D
\end{array}\right],
$$

where $D$ is a constant.

CASE $I I I$ : This case is similar to CASE $I I$.

A Proposition, a Corollary and a Remark similar to Proposition 1.1, Corollary 1.1 and Remark 1.2 will be stated, without proofs, for each of the remaining distributions listed in Section 1.

Proposition 1.3. Let $X: \Omega \rightarrow(0, \infty)$ be a continuous random variable and let $q_{1}(x)=\left\{1-\left[1-\exp \left(-\frac{\theta}{\gamma}\left(e^{\gamma x}-1\right)\right)\right]^{c}\right\}^{1-b}$ 
and $q_{2}(x)=q_{1}(x)\left[1-\exp \left(-\frac{\theta}{\gamma}\left(e^{\gamma x}-1\right)\right)\right]^{a c}$ for $x>0$. Then, the random variable $X$ has $p d f(4)$ if and only if the function $\xi$ defined in Theorem 1 is of the form

$$
\xi(x)=\frac{1}{2}\left\{1+\left[1-\exp \left(-\frac{\theta}{\gamma}\left(e^{\gamma x}-1\right)\right)\right]^{a c}\right\}, \quad x>0 .
$$

Corollary 1.3. Let $X: \Omega \rightarrow(0, \infty)$ be a continuous random variable and let $q_{1}(x)$ be as in Proposition 1.3 . The random variable $X$ has $p d f(4)$ if and only if there exist functions $q_{2}$ and $\xi$ defined in Theorem 1 satisfying the following differential equation

$$
\frac{\xi^{\prime}(x) q_{1}(x)}{\xi(x) q_{1}(x)-q_{2}(x)}=\frac{a c \theta e^{\gamma x} \exp \left(-\frac{\theta}{\gamma}\left(e^{\gamma x}-1\right)\right)\left[1-\exp \left(-\frac{\theta}{\gamma}\left(e^{\gamma x}-1\right)\right)\right]^{a c-1}}{1-\left[1-\exp \left(-\frac{\theta}{\gamma}\left(e^{\gamma x}-1\right)\right)\right]^{a c}}, \quad x>0 .
$$

Remark 1.4. The general solution of the differential equation in Corollary 1.3 is

$$
\begin{aligned}
\xi(x)= & \left\{1-\left[1-\exp \left(-\frac{\theta}{\gamma}\left(e^{\gamma x}-1\right)\right)\right]^{a c}\right\}^{-1} \times \\
& {\left[\begin{array}{c}
-\int a c \theta e^{\gamma x} \exp \left(-\frac{\theta}{\gamma}\left(e^{\gamma x}-1\right)\right) \times \\
{\left[1-\exp \left(-\frac{\theta}{\gamma}\left(e^{\gamma x}-1\right)\right)\right]^{a c-1}\left(q_{1}(x)\right)^{-1} q_{2}(x) d x+D}
\end{array}\right], }
\end{aligned}
$$

where $D$ is a constant.

Proposition 1.4. Let $X: \Omega \rightarrow(0, \infty)$ be a continuous random variable and let

$q_{1}(x)=\frac{\left.\left.\left\{1-\left[1-e^{-\left(\theta x+\gamma x^{\beta}\right.}\right)\right]^{\alpha a}\left[1+\lambda-\lambda\left(1-e^{-\left(\theta x+\gamma x^{\beta}\right.}\right)\right)^{\alpha}\right]^{a}\right\}^{1-b}\left(1-e^{-\left(\theta x+\gamma \beta^{\beta}\right)}\right)^{\alpha(1-a)}}{\left.\left[1+\lambda-2 \lambda\left(1-e^{-\left(\theta x+\gamma x^{\beta}\right.}\right)\right)^{\alpha}\right]}$ and $q_{2}(x)=q_{1}(x)\left[1+\lambda-\lambda\left(1-e^{-\left(\theta x+\gamma x^{\beta}\right)}\right)^{\alpha}\right]^{a}$ for $x>0$.

Then, the random variable $X$ has $p d f(6)$ if and only if the function $\xi$ defined in Theorem 1 is of the form

$$
\xi(x)=\frac{1}{2}\left\{\left[1+\lambda-\lambda\left(1-e^{-\left(\theta x+\gamma x^{\beta}\right)}\right)^{\alpha}\right]^{a}+1\right\}, \quad x>0 .
$$

Corollary 1.4. Let $X: \Omega \rightarrow(0, \infty)$ be a continuous random variable and let $q_{1}(x)$ be as in Proposition 1.4 . The random variable $X$ has $p d f(6)$ if and only if there exist functions $q_{2}$ and $\xi$ defined in Theorem 1 satisfying the following differential equation

$$
\begin{aligned}
& \frac{\xi^{\prime}(x) q_{1}(x)}{\xi(x) q_{1}(x)-q_{2}(x)} \\
& =\frac{a b \alpha\left(\theta+\gamma \beta x^{\beta-1}\right) e^{-\left(\theta x+\gamma x^{\beta}\right)}\left(1-e^{-\left(\theta x+\gamma x^{\beta}\right)}\right)^{\alpha-1} \times}{\left[1+\lambda-2 \lambda\left(1-e^{-\left(\theta x+\gamma x^{\beta}\right)}\right)^{\alpha}\right]\left[1+\lambda-\lambda\left(1-e^{-\left(\theta x+\gamma x^{\beta}\right)}\right)^{\alpha}\right]^{a-1}}, \quad x>0 . \\
& {\left[1+\lambda-\lambda\left(1-e^{-\left(\theta x+\gamma x^{\beta}\right)}\right)^{\alpha}\right]^{a}-1}
\end{aligned}
$$

Remark 1.5. The general solution of the differential equation in Corollary 1.4 is

$$
\begin{aligned}
& \xi(x)=\left\{\left[1+\lambda-\lambda\left(1-e^{-\left(\theta x+\gamma x^{\beta}\right)}\right)^{\alpha}\right]^{a}-1\right\}^{-1} \times \\
& {\left[\begin{array}{c}
-\int a b \alpha\left(\theta+\gamma \beta x^{\beta-1}\right) e^{-\left(\theta x+\gamma x^{\beta}\right)}\left(1-e^{-\left(\theta x+\gamma x^{\beta}\right)}\right)^{\alpha-1} \times \\
{\left[1+\lambda-2 \lambda\left(1-e^{-\left(\theta x+\gamma x^{\beta}\right)}\right)^{\alpha}\right]\left[1+\lambda-\lambda\left(1-e^{-\left(\theta x+\gamma x^{\beta}\right)}\right)^{\alpha}\right]^{a-1}\left(q_{1}(x)\right)^{-1} q_{2}(x)+D}
\end{array}\right],}
\end{aligned}
$$

where $D$ is a constant.

Proposition 1.5. Let $X: \Omega \rightarrow \mathbb{R}$ be a continuous random variable and let $q_{1}(x) \equiv 1$ and $q_{2}(x)=e^{\frac{\theta}{\gamma}\left\{1-(\bar{G}(x ; \eta))^{-\gamma}\right\}}$ for $x \in \mathbb{R}$. Then, the random variable $X$ has $p d f(12)$ if and only if the function $\xi$ defined in Theorem 1 is of the form

$$
\xi(x)=\frac{1}{2} e^{\frac{\theta}{\gamma}\left\{1-(\bar{G}(x ; \eta))^{-\gamma}\right\}}, \quad x \in \mathbb{R} .
$$


Corollary 1.5. Let $X: \Omega \rightarrow \mathbb{R}$ be a continuous random variable and let $q_{1}(x)$ be as in Proposition 1.5. The random variable $X$ has $p d f(12)$ if and only if there exist functions $q_{2}$ and $\xi$ defined in Theorem 1 satisfying the following differential equation

$$
\frac{\xi^{\prime}(x)}{\xi(x)-q_{2}(x)}=\theta g(x ; \eta)(\bar{G}(x ; \eta))^{-\gamma-1}, \quad x \in \mathbb{R} .
$$

Remark 1.6. The general solution of the differential equation in Corollary 1.5 is

$$
\xi(x)=e^{-\frac{\theta}{\gamma}\left\{1-(\bar{G}(x ; \eta))^{-\gamma}\right\}}\left[-\int \theta g(x ; \eta) e^{\frac{\theta}{\gamma}\left\{1-(\bar{G}(x ; \eta))^{-\gamma}\right\}}\left(q_{1}(x)\right)^{-1} q_{2}(x)+D\right],
$$

where $D$ is a constant.

Proposition 1.6. Let $X: \Omega \rightarrow(0, \infty)$ be a continuous random variable and let $q_{1}(x)=\left[1+\lambda-\frac{2 \lambda \theta\left(1-e^{-(\alpha x)^{\beta}}\right)}{1+(\theta-1)\left(1-e^{-(\alpha x)^{\beta}}\right)}\right]^{-1}$ and $q_{2}(x)=q_{1}(x)\left[1+(\theta-1)\left(1-e^{-(\alpha x)^{\beta}}\right)\right]^{-1}$ for $x>0$. The random variable $X$ has $p d f(16)$ if and only if the function $\xi$ defined in Theorem1 has the form

$$
\xi(x)=\frac{1}{2}\left\{\left[1+(\theta-1)\left(1-e^{-(\alpha x)^{\beta}}\right)\right]^{-1}+\theta^{-1}\right\}, \quad x>0 .
$$

Corollary 1.6. Let $X: \Omega \rightarrow(0, \infty)$ be a continuous random variable and let $q_{1}(x)$ be as in Proposition 1.6. The random variable $X$ has $p d f(16)$ if and only if there exist functions $q_{2}$ and $\xi$ defined in Theorem1 satisfying the following differential equation

$$
\frac{\xi^{\prime}(x) q_{1}(x)}{\xi(x) q_{1}(x)-q_{2}(x)}=\frac{(\theta-1) \beta \alpha^{\beta} x^{\beta-1} e^{-(\alpha x)^{\beta}}\left[1+(\theta-1)\left(1-e^{-(\alpha x)^{\beta}}\right)\right]^{-2}}{\left[1+(\theta-1)\left(1-e^{-(\alpha x)^{\beta}}\right)\right]^{-1}-\theta^{-1}}, \quad x>0 .
$$

Remark 1.7. The general solution of the differential equation in Corollary 1.6 is

$$
\xi(x)=\left\{\left[1+(\theta-1)\left(1-e^{-(\alpha x)^{\beta}}\right)\right]^{-1}-\theta^{-1}\right\}\left[\begin{array}{c}
-\int \frac{(\theta-1) \beta \alpha^{\beta} x^{\beta-1} e^{-\left(\alpha x x^{\beta}\right.}\left[1+(\theta-1)\left(1-e^{-(\alpha x \beta}\right)\right]^{-2}}{\left\{\left[1+(\theta-1)\left(1-e^{-(\alpha x)^{\beta}}\right)\right]^{-1}-\theta^{-1}\right\}^{2}} \\
\left(q_{1}(x)\right)^{-1} q_{2}(x) d x+D
\end{array}\right],
$$

where $D$ is a constant.

Remark 1.8. Proposition 1.6, Corollary 1.6 and Remark 1.7 were mentioned incorrectly in Nofal et al. (2017).

Proposition 1.7. Let $X: \Omega \rightarrow(0, \infty)$ be a continuous random variable and let $q_{1}(x)=\left[\delta^{\theta}+\left(1-e^{-\eta x}\right)^{\theta}\right]^{2}$ and $q_{2}(x)=$ $q_{1}(x)\left(1-e^{-\eta x}\right)^{\theta}$ for $x>0$. The random variable $X$ has $p d f(18)$ if and only if the function $\xi$ defined in Theorem1 has the form

$$
\xi(x)=\frac{1}{2}\left\{1+\left(1-e^{-\eta x}\right)^{\theta}\right\}, \quad x>0 .
$$

Corollary 1.7. Let $X: \Omega \rightarrow(0, \infty)$ be a continuous random variable and let $q_{1}(x)$ be as in Proposition 1.7. The random variable $X$ has $p d f(18)$ if and only if there exist functions $q_{2}$ and $\xi$ defined in Theorem1 satisfying the following differential equation

$$
\frac{\xi^{\prime}(x) q_{1}(x)}{\xi(x) q_{1}(x)-q_{2}(x)}=\frac{\eta \theta e^{-\eta x}\left(1-e^{-\eta x}\right)^{\theta-1}}{1-\left(1-e^{-\eta x}\right)^{\theta}}, \quad x>0 .
$$

Remark 1.9. The general solution of the differential equation in Corollary 1.7 is

$$
\xi(x)=\left[1-\left(1-e^{-\eta x}\right)^{\theta}\right]^{-1}\left[-\int \eta \theta e^{-\eta x}\left(1-e^{-\eta x}\right)^{\theta-1}\left(q_{1}(x)\right)^{-1} q_{2}(x) d x+D\right],
$$

where $D$ is a constant.

Proposition 1.8. Let $X: \Omega \rightarrow(0, \infty)$ be a continuous random variable and let $q_{1}(x)=\left[1+[G(x ; \zeta)]^{\lambda}\right]^{2}$ and $q_{2}(x)=$ $q_{1}(x)[G(x ; \zeta)]^{\lambda}$ for $x>0$. The random variable $X$ has $p d f(20)$ if and only if the function $\xi$ defined in Theorem 1 has the form

$$
\xi(x)=\frac{1}{2}\left\{1+[G(x ; \zeta)]^{\lambda}\right\}, \quad x>0 .
$$


Corollary 1.8. Let $X: \Omega \rightarrow(0, \infty)$ be a continuous random variable and let $q_{1}(x)$ be as in Proposition 1.8 . The random variable $X$ has $p d f$ (20) if and only if there exist functions $q_{2}$ and $\xi$ defined in Theorem 1 satisfying the following differential equation

$$
\frac{\xi^{\prime}(x) q_{1}(x)}{\xi(x) q_{1}(x)-q_{2}(x)}=\frac{\lambda g(x ; \zeta)[G(x ; \zeta)]^{\lambda-1}}{1-[G(x ; \zeta)]^{\lambda}}, \quad x>0
$$

Remark 1.10. The general solution of the differential equation in Corollary 1.8 is

$$
\xi(x)=\left[1-[G(x ; \zeta)]^{\lambda}\right]^{-1}\left[-\int \lambda g(x ; \zeta)[G(x ; \zeta)]^{\lambda-1}\left(q_{1}(x)\right)^{-1} q_{2}(x) d x+D\right]
$$

where $D$ is a constant.

Proposition 1.9. Let $X: \Omega \rightarrow(0, \infty)$ be a continuous random variable and let $q_{1}(x)=\left\{1-\lambda+2 \lambda\left[1-\exp \left\{-\frac{\alpha}{x}-\gamma\left(\frac{1}{x}\right)^{\beta}\right\}^{\phi}\right\}^{-1}\right.$ and $q_{2}(x)=q_{1}(x)\left[1-\exp \left\{-\frac{\alpha}{x}-\gamma\left(\frac{1}{x}\right)^{\beta}\right\}\right]$ for $x>0$. The random variable $X$ has $p d f(22)$ if and only if the function $\xi$ defined in Theorem1 has the form

$$
\xi(x)=\frac{\phi}{\phi+1}\left[1-\exp \left\{-\frac{\alpha}{x}-\gamma\left(\frac{1}{x}\right)^{\beta}\right\}\right], \quad x>0 .
$$

Corollary 1.9. Let $X: \Omega \rightarrow(0, \infty)$ be a continuous random variable and let $q_{1}(x)$ be as in Proposition 1.9 . The random variable $X$ has $p d f(22)$ if and only if there exist functions $q_{2}$ and $\xi$ defined in Theorem 1 satisfying the following differential equation

$$
\frac{\xi^{\prime}(x) q_{1}(x)}{\xi(x) q_{1}(x)-q_{2}(x)}=\frac{\phi\left\{\frac{\alpha}{x^{2}}+\beta \gamma\left(\frac{1}{x}\right)^{\beta+1}\right\} \exp \left\{-\frac{\alpha}{x}-\gamma\left(\frac{1}{x}\right)^{\beta}\right\}}{1-\exp \left\{-\frac{\alpha}{x}-\gamma\left(\frac{1}{x}\right)^{\beta}\right\}}, \quad x>0 .
$$

Remark 1.11. The general solution of the differential equation in Corollary 1.9 is

$$
\xi(x)=\left[1-\exp \left\{-\frac{\alpha}{x}-\gamma\left(\frac{1}{x}\right)^{\beta}\right\}\right]^{-1}\left[\begin{array}{c}
-\int \phi\left\{\frac{\alpha}{x^{2}}+\beta \gamma\left(\frac{1}{x}\right)^{\beta+1}\right\} \times \\
\exp \left\{-\frac{\alpha}{x}-\gamma\left(\frac{1}{x}\right)^{\beta}\right\}\left(q_{1}(x)\right)^{-1} q_{2}(x) d x+D
\end{array}\right]
$$

where $D$ is a constant.

Proposition 1.10. Let $X: \Omega \rightarrow \mathbb{R}$ be a continuous random variable and let $q_{1}(x) \equiv 1$ and $q_{2}(x)=\exp [\theta G(x ; \eta)]$ for $x \in \mathbb{R}$. The random variable $X$ has $p d f(24)$ if and only if the function $\xi$ defined in Theorem 1 has the form

$$
\xi(x)=\frac{1}{2}\left\{e^{\theta}+\exp [\theta G(x ; \eta)]\right\}, \quad x \in \mathbb{R} .
$$

Corollary 1.10. Let $X: \Omega \rightarrow \mathbb{R}$ be a continuous random variable and let $q_{1}(x)$ be as in Proposition 1.10 . The random variable $X$ has $p d f(24)$ if and only if there exist functions $q_{2}$ and $\xi$ defined in Theorem 1 satisfying the following differential equation

$$
\frac{\xi^{\prime}(x)}{\xi(x)-q_{2}(x)}=\frac{\theta g(x ; \eta) \exp [\theta G(x ; \eta)]}{e^{\theta}-\exp [\theta G(x ; \eta)]}, \quad x>0 .
$$

Remark 1.12. The general solution of the differential equation in Corollary 1.10 is

$$
\xi(x)=\left\{e^{\theta}-\exp [\theta G(x ; \eta)]\right\}^{-1}\left[-\int \theta g(x ; \eta) \exp [\theta G(x ; \eta)] q_{2}(x) d x+D\right],
$$

where $D$ is a constant.

Proposition 1.11. Let $X: \Omega \rightarrow(0, \infty)$ be a continuous random variable and let $q_{1}(x)=\left\{a(1+\lambda)-\lambda(a+b)\left[1-e^{-(\alpha x)^{\beta}}\right]^{b}\right\}^{-1}$ and $q_{2}(x)=q_{1}(x)\left[1-e^{-(\alpha x)^{\beta}}\right]^{a}$ for $x>0$. The random variable $X$ has $p d f(26)$ if and only if the function $\xi$ defined in Theorem1 has the form

$$
\xi(x)=\frac{1}{2}\left\{1+\left[1-e^{-(\alpha x)^{\beta}}\right]^{a}\right\}, \quad x>0 .
$$


Corollary 1.11. Let $X: \Omega \rightarrow \mathbb{R}$ be a continuous random variable and let $q_{1}(x)$ be as in Proposition 1.11 . The random variable $X$ has $p d f(26)$ if and only if there exist functions $q_{2}$ and $\xi$ defined in Theorem 1 satisfying the following differential equation

$$
\frac{\xi^{\prime}(x) q_{1}(x)}{\xi(x) q_{1}(x)-q_{2}(x)}=\frac{a \beta \alpha^{\beta} x^{\beta-1} e^{-(\alpha x)^{\beta}}\left[1-e^{-(\alpha x)^{\beta}}\right]^{a-1}}{1-\left[1-e^{-(\alpha x)^{\beta}}\right]^{a}}, \quad x>0 .
$$

Remark 1.13. The general solution of the differential equation in Corollary 1.11 is

$$
\xi(x)=\left\{1-\left[1-e^{-(\alpha x)^{\beta}}\right]^{a}\right\}^{-1}\left[\begin{array}{c}
-\int a \beta \alpha^{\beta} x^{\beta-1} e^{-(\alpha x)^{\beta}} \times \\
{\left[1-e^{-(\alpha x)^{\beta}}\right]^{a-1}\left(q_{1}(x)\right)^{-1} q_{2}(x) d x+D}
\end{array}\right],
$$

where $D$ is a constant.

Proposition 1.12. Let $X: \Omega \rightarrow \mathbb{R}$ be a continuous random variable and let $q_{1}(x)=\exp \left\{\lambda\left\{1-[\bar{G}(x ; \psi)]^{a}\right\}^{b}\right\}$ and $q_{2}(x)=q_{1}(x)\left\{1-[\bar{G}(x ; \psi)]^{a}\right\}^{b}$ for $x \in \mathbb{R}$. The random variable $X$ has $p d f(28)$ if and only if the function $\xi$ defined in Theorem1 has the form

$$
\xi(x)=\frac{1}{2}\left\{1+\left\{1-[\bar{G}(x ; \psi)]^{a}\right\}^{b}\right\}, \quad x \in \mathbb{R} .
$$

Corollary 1.12. Let $X: \Omega \rightarrow \mathbb{R}$ be a continuous random variable and let $q_{1}(x)$ be as in Proposition 1.12 . The random variable $X$ has $p d f(28)$ if and only if there exist functions $q_{2}$ and $\xi$ defined in Theorem 1 satisfying the following differential equation

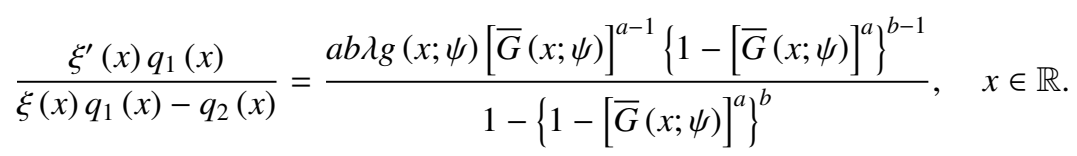

Remark 1.14. The general solution of the differential equation in Corollary 1.12 is

$$
\xi(x)=\left\{1-\left\{1-[\bar{G}(x ; \psi)]^{a}\right\}^{b}\right\}^{-1}\left[\begin{array}{c}
-\int a b \lambda g(x ; \psi)[\bar{G}(x ; \psi)]^{a-1}\left\{1-[\bar{G}(x ; \psi)]^{a}\right\}^{b-1} \\
\times\left(q_{1}(x)\right)^{-1} q_{2}(x) d x+D
\end{array}\right]
$$

where $D$ is a constant.

Proposition 1.13. Let $X: \Omega \rightarrow(0, \infty)$ be a continuous random variable and let $q_{1}(x)=\left[1-\exp \left(-\theta x^{-2}\right)\right]^{1-b}$ and $q_{2}(x)=q_{1}(x) \exp \left(-\theta x^{-2}\right)$ for $x>0$. The random variable $X$ has $p d f$ (30) if and only if the function $\xi$ defined in Theorem1 has the form

$$
\xi(x)=\frac{1}{2}\left\{1+\exp \left(-\theta x^{-2}\right)\right\}, \quad x>0 .
$$

Corollary 1.13. Let $X: \Omega \rightarrow(0, \infty)$ be a continuous random variable and let $q_{1}(x)$ be as in Proposition 1.13 . The random variable $X$ has $p d f(30)$ if and only if there exist functions $q_{2}$ and $\xi$ defined in Theorem 1 satisfying the following differential equation

$$
\frac{\xi^{\prime}(x) q_{1}(x)}{\xi(x) q_{1}(x)-q_{2}(x)}=\frac{a \theta x^{-3} \exp \left(-\theta x^{-2}\right)}{1-\exp \left(-\theta x^{-2}\right)}, \quad x>0 .
$$

Remark 1.15. The general solution of the differential equation in Corollary 1.13 is

$$
\xi(x)=\left\{1-\exp \left(-\theta x^{-2}\right)\right\}^{-1}\left[-\int a \theta x^{-3} \exp \left(-\theta x^{-2}\right)\left(q_{1}(x)\right)^{-1} q_{2}(x) d x+D\right], \text { where }
$$

Disaconstant.

Proposition 1.14. Let $\mathrm{X}: \Omega \rightarrow(0, \infty)$ be a continuous random variable and let $q_{1}(x)=\sqrt{1-\exp \left(-\theta x^{-2}\right)}$ and $q_{2}(x)=$ $q_{1}(x) \exp \left(-\frac{\theta}{2} x^{-2}\right)$ for $x>0$. The random variable $X$ has $p d f$ (32) if and only if the function $\xi$ defined in Theorem1 has the form

$$
\xi(x)=\frac{1}{2}\left\{1+\exp \left(-\frac{\theta}{2} x^{-2}\right)\right\}, \quad x>0 .
$$

Corollary 1.14. Let $X: \Omega \rightarrow(0, \infty)$ be a continuous random variable and let $q_{1}(x)$ be as in Proposition 1.14 . The random variable $X$ has $p d f(32)$ if and only if there exist functions $q_{2}$ and $\xi$ defined in Theorem 1 satisfying the following differential equation

$$
\frac{\xi^{\prime}(x) q_{1}(x)}{\xi(x) q_{1}(x)-q_{2}(x)}=\frac{\theta x^{-3} \exp \left(-\frac{\theta}{2} x^{-2}\right)}{1-\exp \left(-\frac{\theta}{2} x^{-2}\right)}, \quad x>0 .
$$


Remark 1.16. The general solution of the differential equation in Corollary 1.14 is

$$
\xi(x)=\left\{1-\exp \left(-\frac{\theta}{2} x^{-2}\right)\right\}^{-1}\left[-\int \theta x^{-3} \exp \left(-\frac{\theta}{2} x^{-2}\right)\left(q_{1}(x)\right)^{-1} q_{2}(x) d x+D\right],
$$

where $D$ is a constant.

Proposition 1.15. Let $X: \Omega \rightarrow(0, \infty)$ be a continuous random variable and let $q_{1}(x)=\left(e^{\lambda}-e^{\lambda e^{-\beta x^{\alpha}}}\right)^{1-a}$ and $q_{2}(x)=$ $q_{1}(x)\left(e^{\lambda e^{-\beta x^{\alpha}}}-1\right)$ for $x>0$. The random variable $X$ has $p d f(34)$ if and only if the function $\xi$ defined in Theorem1 has the form

$$
\xi(x)=\frac{b}{b+1}\left(e^{\lambda e^{-\beta x^{\alpha}}}-1\right), \quad x>0 .
$$

Corollary 1.15. Let $X: \Omega \rightarrow(0, \infty)$ be a continuous random variable and let $q_{1}(x)$ be as in Proposition 1.15 . The random variable $X$ has $p d f$ (34) if and only if there exist functions $q_{2}$ and $\xi$ defined in Theorem1 satisfying the following differential equation

$$
\frac{\xi^{\prime}(x) q_{1}(x)}{\xi(x) q_{1}(x)-q_{2}(x)}=\frac{b \alpha \beta \lambda x^{\alpha-1} e^{\lambda e^{-\beta x^{\alpha}}}}{e^{\lambda e^{-\beta x^{\alpha}}}-1}, \quad x>0
$$

Remark 1.17. The general solution of the differential equation in Corollary 1.15 is

$$
\xi(x)=\left\{e^{\lambda e^{-\beta x^{\alpha}}}-1\right\}^{-1}\left[-\int b \alpha \beta \lambda x^{\alpha-1} e^{\lambda e^{-\beta x^{\alpha}}}\left(q_{1}(x)\right)^{-1} q_{2}(x) d x+D\right],
$$

where $D$ is a constant.

Proposition 1.16. Let $X: \Omega \rightarrow(0, \infty)$ be a continuous random variable and let $q_{1}(x)=\left(\alpha x^{\beta} e^{\lambda x}\right)^{1-a}$ and $q_{2}(x)=$ $q_{1}(x) e^{\lambda x-\alpha x^{\beta} e^{\lambda x}}$ for $x>0$. The random variable $X$ has $p d f(36)$ if and only if the function $\xi$ defined in Theorem1 has the form

$$
\xi(x)=\frac{1}{2} e^{-\alpha x^{\beta} e^{\lambda x}}, \quad x>0 .
$$

Corollary 1.16. Let $X: \Omega \rightarrow(0, \infty)$ be a continuous random variable and let $q_{1}(x)$ be as in Proposition 1.16 . The random variable $X$ has $p d f(36)$ if and only if there exist functions $q_{2}$ and $\xi$ defined in Theorem 1 satisfying the following differential equation

$$
\frac{\xi^{\prime}(x) q_{1}(x)}{\xi(x) q_{1}(x)-q_{2}(x)}=\alpha x^{\beta-1}(\beta+x) e^{\lambda x}, \quad x>0 .
$$

Remark 1.18. The general solution of the differential equation in Corollary 1.16 is

$$
\xi(x)=e^{\alpha x^{\beta} e^{\lambda x}}\left[-\int \alpha x^{\beta-1}(\beta+x) e^{\lambda x-\alpha x^{\beta} e^{\lambda x}}\left(q_{1}(x)\right)^{-1} q_{2}(x) d x+D\right],
$$

where $D$ is a constant.

Proposition 1.17. Let $X: \Omega \rightarrow(0, \infty)$ be a continuous random variable and let $q_{1}(x)=\left[1+\lambda-2 \lambda\left(1+\alpha x^{-\theta}\right)^{-\beta}\right]^{-1}$ and $q_{2}(x)=q_{1}(x)\left(1+\alpha x^{-\theta}\right)^{-\beta}$ for $x>0$. The random variable $X$ has $p d f(38)$ if and only if the function $\xi$ defined in Theorem1 has the form

$$
\xi(x)=\frac{1}{2}\left\{1+\left(1+\alpha x^{-\theta}\right)^{-\beta}\right\}, \quad x>0 .
$$

Corollary 1.17. Let $X: \Omega \rightarrow(0, \infty)$ be a continuous random variable and let $q_{1}(x)$ be as in Proposition 1.17 . The random variable $X$ has $p d f(38)$ if and only if there exist functions $q_{2}$ and $\xi$ defined in Theorem 1 satisfying the following differential equation

$$
\frac{\xi^{\prime}(x) q_{1}(x)}{\xi(x) q_{1}(x)-q_{2}(x)}=\frac{\alpha \beta \theta x^{-\theta-1}\left(1+\alpha x^{-\theta}\right)^{-\beta-1}}{1-\left(1+\alpha x^{-\theta}\right)^{-\beta}}, \quad x>0 .
$$

Remark 1.19. The general solution of the differential equation in Corollary 1.17 is

$$
\xi(x)=\left\{1-\left(1+\alpha x^{-\theta}\right)^{-\beta}\right\}^{-1}\left[-\int \alpha \beta \theta x^{-\theta-1}\left(1+\alpha x^{-\theta}\right)^{-\beta-1}\left(q_{1}(x)\right)^{-1} q_{2}(x) d x+D\right],
$$

where $D$ is a constant.

Proposition 1.18. Let $X: \Omega \rightarrow \mathbb{R}$ be a continuous random variable and let $q_{1}(x)=\left\{1-e^{\left.\frac{\theta}{\gamma}\left(1-(\bar{G}(x ; \eta))^{-\gamma}\right)\right\}^{1-\alpha}}\right.$ and 
$q_{2}(x)=q_{1}(x) e^{\frac{\theta}{\gamma}\left(1-(\bar{G}(x ; \eta))^{-\gamma}\right)}$ for $x \in \mathbb{R}$. The random variable $X$ has $p d f(40)$ if and only if the function $\xi$ defined in Theorem1 has the form

$$
\xi(x)=\frac{1}{2} e^{\frac{\theta}{\gamma}\left(1-(\bar{G}(x ; \eta))^{-\gamma}\right)}, \quad x \in \mathbb{R} .
$$

Corollary 1.18. Let $X: \Omega \rightarrow \mathbb{R}$ be a continuous random variable and let $q_{1}(x)$ be as in Proposition 1.18. The random variable $X$ has $p d f(40)$ if and only if there exist functions $q_{2}$ and $\xi$ defined in Theorem1 satisfying the following differential equation

$$
\frac{\xi^{\prime}(x) q_{1}(x)}{\xi(x) q_{1}(x)-q_{2}(x)}=\theta g(x ; \eta)(\bar{G}(x ; \eta))^{-\gamma-1}, \quad x \in \mathbb{R} .
$$

Remark 1.20. The general solution of the differential equation in Corollary 1.18 is

$$
\xi(x)=e^{-\frac{\theta}{\gamma}\left(1-(\bar{G}(x ; \eta))^{-\gamma}\right)}\left[-\int \theta g(x ; \eta)(\bar{G}(x ; \eta))^{-\gamma-1} e^{\frac{\theta}{\gamma}\left(1-(\bar{G}(x ; \eta))^{-\gamma}\right)}\left(q_{1}(x)\right)^{-1} q_{2}(x) d x+D\right],
$$

where $D$ is a constant.

Proposition 1.19. Let $X: \Omega \rightarrow(0, \infty)$ be a continuous random variable and let $q_{1}(x)=\left\{\left[1-\left(1+\frac{\lambda}{1+\lambda} x^{\beta}\right) e^{-\lambda x^{\beta}}\right]^{\alpha}+\left(1+\frac{\lambda}{1+\lambda} x^{\beta}\right) e^{-\lambda x^{\beta}}\right\}^{2}$ and $q_{2}(x)=q_{1}(x)\left[1-\left(1+\frac{\lambda}{1+\lambda} x^{\beta}\right) e^{-\lambda x^{\beta}}\right]^{\alpha}$ for $x>0$. The random variable $X$ has $p d f(42)$ if and only if the function $\xi$ defined in Theorem 1 has the form

$$
\xi(x)=\frac{1}{2}\left\{1+\left[1-\left(1+\frac{\lambda}{1+\lambda} x^{\beta}\right) e^{-\lambda x^{\beta}}\right]\right\}, \quad x>0 .
$$

Corollary 1.19. Let $X: \Omega \rightarrow(0, \infty)$ be a continuous random variable and let $q_{1}(x)$ be as in Proposition 1.19. The random variable $X$ has $p d f(42)$ if and only if there exist functions $q_{2}$ and $\xi$ defined in Theorem1 satisfying the following differential equation

$$
\frac{\xi^{\prime}(x) q_{1}(x)}{\xi(x) q_{1}(x)-q_{2}(x)}=\frac{\alpha \beta \lambda^{2} x^{\beta-1}\left(1+x^{\beta}\right) e^{-\lambda x^{\beta}}\left[1-\left(1+\frac{\lambda}{1+\lambda} x^{\beta}\right) e^{-\lambda x^{\beta}}\right]^{\alpha-1}}{1-\left[1-\left(1+\frac{\lambda}{1+\lambda} x^{\beta}\right) e^{-\lambda x^{\beta}}\right]^{\alpha}}, \quad x>0 .
$$

Remark 1.21. The general solution of the differential equation in Corollary 1.19 is

$$
\begin{aligned}
\xi(x) & =\left\{1-\left[1-\left(1+\frac{\lambda}{1+\lambda} x^{\beta}\right) e^{-\lambda x^{\beta}}\right]^{\alpha}\right\}^{-1} \times \\
& {\left[-\int \alpha \beta \lambda^{2} x^{\beta-1}\left(1+x^{\beta}\right) e^{-\lambda x^{\beta}} \times\left[1-\left(1+\frac{\lambda}{1+\lambda} x^{\beta}\right) e^{-\lambda x^{\beta}}\right]^{\alpha-1}\left(q_{1}(x)\right)^{-1} q_{2}(x) d x+D\right], }
\end{aligned}
$$

where $D$ is a constant.

Proposition 1.20. Let $X: \Omega \rightarrow \mathbb{R}$ be a continuous random variable and let $q_{1}(x)=e^{-\alpha e^{-e^{x}}}$ and $q_{2}(x)=q_{1}(x) e^{-e^{x}}$ for $x \in \mathbb{R}$. The random variable $X$ has $p d f(44)$ if and only if the function $\xi$ defined in Theorem 1 has the form

$$
\xi(x)=\frac{1}{2} e^{-e^{x}}, \quad x \in \mathbb{R} .
$$

Corollary 1.20. Let $X: \Omega \rightarrow \mathbb{R}$ be a continuous random variable and let $q_{1}(x)$ be as in Proposition 1.20. The random variable $X$ has $p d f(44)$ if and only if there exist functions $q_{2}$ and $\xi$ defined in Theorem1 satisfying the following differential equation

$$
\frac{\xi^{\prime}(x) q_{1}(x)}{\xi(x) q_{1}(x)-q_{2}(x)}=e^{x}, \quad x \in \mathbb{R} .
$$

Remark 1.22. The general solution of the differential equation in Corollary 1.20 is

$$
\xi(x)=e^{e^{x}}\left[-\int e^{x} e^{-e^{x}}\left(q_{1}(x)\right)^{-1} q_{2}(x) d x+D\right]
$$

where $D$ is a constant.

Proposition 1.21. Let $X: \Omega \rightarrow \mathbb{R}$ be a continuous random variable and let $q_{1}(x)=\frac{\phi(\lambda x)\left\{\left[\Phi_{S N}(x ; \lambda)\right]^{\alpha}+\left[\bar{\Phi}_{S N}(x ; \lambda)\right]^{\alpha}\right\}^{2}}{\phi(x)\left[\Phi_{S N}(x ; \lambda)\right]^{\alpha-1}\left[\bar{\Phi}_{S N}(x ; \lambda)\right]^{\alpha-1}}$ and $q_{2}(x)=q_{1}(x) \Phi(\lambda x)$ for $x \in \mathbb{R}$. The random variable $X$ has $p d f(46)$ if and only if the function $\xi$ defined in Theorem1 has the form

$$
\xi(x)=\frac{1}{2}\left\{1+[\Phi(\lambda x)]^{2}\right\}, \quad x \in \mathbb{R} .
$$


Corollary 1.21. Let $X: \Omega \rightarrow \mathbb{R}$ be a continuous random variable and let $q_{1}(x)$ be as in Proposition 1.21. The random variable $X$ has $p d f(46)$ if and only if there exist functions $q_{2}$ and $\xi$ defined in Theorem1 satisfying the following differential equation

$$
\frac{\xi^{\prime}(x) q_{1}(x)}{\xi(x) q_{1}(x)-q_{2}(x)}=\frac{2 \lambda \phi(\lambda x) \Phi(\lambda x)}{1-[\Phi(\lambda x)]^{2}}, \quad x \in \mathbb{R} .
$$

Remark 1.23. The general solution of the differential equation in Corollary 1.21 is

$$
\xi(x)=\left\{1-[\Phi(\lambda x)]^{2}\right\}^{-1}\left[-\int 2 \lambda \phi(\lambda x) \Phi(\lambda x)\left(q_{1}(x)\right)^{-1} q_{2}(x) d x+D\right],
$$

where $D$ is a constant.

Proposition 1.22. Let $X: \Omega \rightarrow(0, \infty)$ be a continuous random variable and let $q_{1}(x)=\left(\frac{x}{\alpha}\right)^{\tau(1-k)} \frac{\left(1-\gamma_{1}\left[k,\left(\frac{x}{\alpha}\right)^{\tau}\right]\right)^{1-\lambda}}{\left.\left(1-p\left\{1-\gamma_{1}\left[k, \frac{x}{\alpha}\right)^{\tau}\right]\right\}\right)^{1-3}}$ and $q_{2}(x)=q_{1}(x) \exp \left(-\left(\frac{x}{\alpha}\right)^{\tau}\right)$ for $x>0$. The random variable $X$ has $p d f(48)$ if and only if the function $\xi$ defined in Theorem1 has the form

$$
\xi(x)=\frac{1}{2} \exp \left(-\left(\frac{x}{\alpha}\right)^{\tau}\right), \quad x>0 .
$$

Corollary 1.22. Let $X: \Omega \rightarrow(0, \infty)$ be a continuous random variable and let $q_{1}(x)$ be as in Proposition 1.22. The random variable $X$ has $p d f(48)$ if and only if there exist functions $q_{2}$ and $\xi$ defined in Theorem1 satisfying the following differential equation

$$
\frac{\xi^{\prime}(x) q_{1}(x)}{\xi(x) q_{1}(x)-q_{2}(x)}=\frac{\tau}{\alpha}\left(\frac{x}{\alpha}\right)^{\tau-1}, \quad x>0 .
$$

Remark 1.24. The general solution of the differential equation in Corollary 1.22 is

$$
\xi(x)=\exp \left(\left(\frac{x}{\alpha}\right)^{\tau}\right)\left[-\int \frac{\tau}{\alpha}\left(\frac{x}{\alpha}\right)^{\tau-1} \exp \left(-\left(\frac{x}{\alpha}\right)^{\tau}\right)\left(q_{1}(x)\right)^{-1} q_{2}(x) d x+D\right]
$$

where $D$ is a constant.

Proposition 1.23. Let $X: \Omega \rightarrow(0, \infty)$ be a continuous random variable and let $q_{1}(x)=\frac{\left\{1-\left(\begin{array}{c}(1+\lambda)\left(1-\frac{\mu \theta+1+\theta x}{\mu \theta+1} e^{-\theta x}\right) \\ -\lambda\left(1-\frac{\mu \theta+1+\theta x}{\mu \theta+1} e^{-\theta x}\right)^{2}\end{array}\right]\right\}}{\left.\left[1-\left\{\begin{array}{c}1-\lambda)\left(1-\frac{\mu \theta+1+\theta x}{\mu \theta+1} e^{-\theta x}\right) \\ -\lambda\left(1-\frac{\mu \theta+1+\theta x}{\mu \theta+1} e^{-\theta x}\right)^{2}\end{array}\right]\right\}\right]^{\alpha \beta-1}}$ and $q_{2}(x)=q_{1}(x)\left[1+\lambda-2 \lambda\left(1-\frac{\mu \theta+1+\theta x}{\mu \theta+1} e^{-\theta x}\right)\right]^{2}$ for $x>0$. The random variable $X$ has $p d f(50)$ if and only if the function $\xi$ defined in Theorem 1 has the form

$$
\xi(x)=\frac{1}{2}\left\{\left[1+\lambda-2 \lambda\left(1-\frac{\mu \theta+1+\theta x}{\mu \theta+1} e^{-\theta x}\right)\right]^{2}+(1+\lambda)^{2}\right\}, \quad x>0 .
$$

Corollary 1.23. Let $X: \Omega \rightarrow(0, \infty)$ be a continuous random variable and let $q_{1}(x)$ be as in Proposition 1.23. The random variable $X$ has $p d f(50)$ if and only if there exist functions $q_{2}$ and $\xi$ defined in Theorem1 satisfying the following differential equation

$$
\frac{\xi^{\prime}(x) q_{1}(x)}{\xi(x) q_{1}(x)-q_{2}(x)}=\frac{4 \lambda \theta^{2}(\mu \theta+1)^{-1}(\mu+x) e^{-\theta x}\left(1-\frac{\mu \theta+1+\theta x}{\mu \theta+1} e^{-\theta x}\right)}{\left\{\left[1+\lambda-2 \lambda\left(1-\frac{\mu \theta+1+\theta x}{\mu \theta+1} e^{-\theta x}\right)\right]^{2}-(1+\lambda)^{2}\right\}}, \quad x>0 .
$$

Remark 1.25. The general solution of the differential equation in Corollary 1.23 is

$$
\begin{aligned}
\xi(x) & =\left\{\left[1+\lambda-2 \lambda\left(1-\frac{\mu \theta+1+\theta x}{\mu \theta+1} e^{-\theta x}\right)\right]^{2}-(1+\lambda)^{2}\right\}^{-1} \times \\
& {\left[-\int 4 \lambda \theta^{2}(\mu \theta+1)^{-1}(\mu+x) e^{-\theta x}\left(1-\frac{\mu \theta+1+\theta x}{\mu \theta+1} e^{-\theta x}\right)\left(q_{1}(x)\right)^{-1} q_{2}(x) d x+D\right], }
\end{aligned}
$$

where $D$ is a constant.

Proposition 1.24. Let $X: \Omega \rightarrow \mathbb{R}$ be a continuous random variable and let $q_{1}(x)=\left[1-(\Phi(x))^{\lambda}\right]^{1-\varphi}$ and $q_{2}(x)=$ 
$q_{1}(x)(\Phi(x))^{\lambda}$ for $x \in \mathbb{R}$. The random variable $X$ has $p d f(51)$, for $q=0$, if and only if the function $\xi$ defined in Theorem1 has the form

$$
\xi(x)=\frac{1}{2}\left\{1+(\Phi(x))^{\lambda}\right\}, \quad x \in \mathbb{R} .
$$

Corollary 1.24. Let $X: \Omega \rightarrow \mathbb{R}$ be a continuous random variable and let $q_{1}(x)$ be as in Proposition 1.24. The random variable $X$ has $p d f$ (51), for $q=0$, if and only if there exist functions $q_{2}$ and $\xi$ defined in Theorem1 satisfying the following differential equation

$$
\frac{\xi^{\prime}(x) q_{1}(x)}{\xi(x) q_{1}(x)-q_{2}(x)}=\frac{\lambda \exp \left(-\frac{x^{2}}{2}\right)(\Phi(x))^{\lambda-1}}{\sqrt{2 \pi}\left\{1-(\Phi(x))^{\lambda}\right\}}, \quad x \in \mathbb{R}
$$

Remark 1.26. The general solution of the differential equation in Corollary 1.24 is

$$
\xi(x)=\left\{1-(\Phi(x))^{\lambda}\right\}^{-1}\left[-\int \frac{\lambda}{\sqrt{2 \pi}} \exp \left(-\frac{x^{2}}{2}\right)(\Phi(x))^{\lambda-1}\left(q_{1}(x)\right)^{-1} q_{2}(x) d x+D\right],
$$

where $D$ is a constant.

Proposition 1.25. Let $X: \Omega \rightarrow \mathbb{R}$ be a continuous random variable and let $q_{1}(x)=\frac{q^{-1}\left\{1-e^{q x}\right\}\left\{\left(\gamma_{1}\left[q^{-2}, q^{-2} e^{q x}\right]\right)^{1-\lambda}\right\}}{\left\{1-\left(\gamma_{1}\left[q^{-2}, q^{-2} e^{q x}\right]\right)^{\lambda}\right\}^{\varphi-1}}$ and $q_{2}(x)=q_{1}(x) \exp \left\{q^{-1} x-q^{-2} e^{q x}\right\}$ for $x \in \mathbb{R}$. The random variable $X$ has $p d f(51)$, for $q \neq 0$, if and only if the function $\xi$ defined in Theorem 1 has the form

$$
\xi(x)=\frac{1}{2} \exp \left\{q^{-1}-q^{-2} e^{q x}\right\}, \quad x \in \mathbb{R} .
$$

Corollary 1.25. Let $X: \Omega \rightarrow \mathbb{R}$ be a continuous random variable and let $q_{1}(x)$ be as in Proposition 1.25. The random variable $X$ has $p d f(51)$, for $q \neq 0$, if and only if there exist functions $q_{2}$ and $\xi$ defined in Theorem1 satisfying the following differential equation

$$
\frac{\xi^{\prime}(x) q_{1}(x)}{\xi(x) q_{1}(x)-q_{2}(x)}=\frac{\left[q^{-1}-q^{-1} e^{q x}\right] \exp \left\{q^{-1} x-q^{-2} e^{q x}\right\}}{1-\exp \left\{q^{-1} x-q^{-2} e^{q x}\right\}}, \quad x \in \mathbb{R} .
$$

Remark 1.27. The general solution of the differential equation in Corollary 1.25 is

$$
\xi(x)=\left\{1-\exp \left\{q^{-1} x-q^{-2} e^{q x}\right\}\right\}^{-1}\left[\begin{array}{c}
-\int\left[q^{-1}-q^{-1} e^{q x}\right] \exp \left\{q^{-1} x-q^{-2} e^{q x}\right\} \times \\
\left(q_{1}(x)\right)^{-1} q_{2}(x) d x+D
\end{array}\right],
$$

where $D$ is a constant.

Proposition 1.26. Let $X: \Omega \rightarrow(0,1)$ be a continuous random variable and let $q_{1}(x)=\left\{1-\exp \left[-\left(\frac{x}{1-x}\right)^{\beta}\right]\right\}^{1-\lambda}$ and $q_{2}(x)=q_{1}(x) \exp \left[-\left(\frac{x}{1-x}\right)^{\beta}\right]$ for $x \in(0,1)$. The random variable $X$ has $p d f(53)$, if and only if the function $\xi$ defined in Theorem1 has the form

$$
\xi(x)=\frac{1}{2} \exp \left[-\left(\frac{x}{1-x}\right)^{\beta}\right], \quad x \in(0,1) .
$$

Corollary 1.26. Let $X: \Omega \rightarrow(0,1)$ be a continuous random variable and let $q_{1}(x)$ be as in Proposition 1.26. The random variable $X$ has $p d f(53)$, if and only if there exist functions $q_{2}$ and $\xi$ defined in Theorem 1 satisfying the following differential equation

$$
\frac{\xi^{\prime}(x) q_{1}(x)}{\xi(x) q_{1}(x)-q_{2}(x)}=\beta x^{\beta-1}(1-x)^{-(\beta+1)}, \quad x \in(0,1) .
$$

Remark 1.28. The general solution of the differential equation in Corollary 1.26 is

$$
\xi(x)=\left[\left(\frac{x}{1-x}\right)^{\beta}\right]\left[-\int \frac{\beta x^{\beta-1}}{(1-x)^{\beta+1}} \exp \left[-\left(\frac{x}{1-x}\right)^{\beta}\right]\left(q_{1}(x)\right)^{-1} q_{2}(x) d x+D\right],
$$

where $D$ is a constant.

Proposition 1.27. Let $X: \Omega \rightarrow(0, \infty)$ be a continuous random variable and let $q_{1}(x)=(1+\beta x)^{-1}$ and $q_{2}(x)=$ $q_{1}(x) e^{-\alpha x}$ for $x>0$. The random variable $X$ has $p d f(57)$, if and only if the function $\xi$ defined in Theorem 1 has the form

$$
\xi(x)=\frac{1}{2} e^{-\alpha x}, \quad x>0 .
$$


Corollary 1.27. Let $X: \Omega \rightarrow(0, \infty)$ be a continuous random variable and let $q_{1}(x)$ be as in Proposition 1.27 . The random variable $X$ has $p d f(57)$, if and only if there exist functions $q_{2}$ and $\xi$ defined in Theorem 1 satisfying the following differential equation

$$
\frac{\xi^{\prime}(x) q_{1}(x)}{\xi(x) q_{1}(x)-q_{2}(x)}=\alpha, \quad x>0 .
$$

Remark 1.29. The general solution of the differential equation in Corollary 1.27 is

$$
\xi(x)=e^{\alpha x}\left[-\int \alpha e^{-\alpha x}\left(q_{1}(x)\right)^{-1} q_{2}(x) d x+D\right],
$$

where $D$ is a constant.

Proposition 1.28. Let $X: \Omega \rightarrow(0, \infty)$ be a continuous random variable and let $q_{1}(x)=\left\{1-e^{-\lambda \alpha x}-\frac{\left(1-e^{-\lambda \alpha(\beta+1) x}\right)}{(\beta+1)}\right\}^{-1}$ and $q_{2}(x)=q_{1}(x) e^{-\lambda x}$ for $x>0$. The random variable $X$ has $p d f(59)$, if and only if the function $\xi$ defined in Theorem1 has the form

$$
\xi(x)=\frac{1}{2} e^{-\lambda x}, \quad x>0
$$

Corollary 1.28. Let $X: \Omega \rightarrow(0, \infty)$ be a continuous random variable and let $q_{1}(x)$ be as in Proposition 1.28 . The random variable $X$ has $p d f(59)$, if and only if there exist functions $q_{2}$ and $\xi$ defined in Theorem 1 satisfying the following differential equation

$$
\frac{\xi^{\prime}(x) q_{1}(x)}{\xi(x) q_{1}(x)-q_{2}(x)}=\lambda, \quad x>0 .
$$

Remark 1.30. The general solution of the differential equation in Corollary 1.27 is

$$
\xi(x)=e^{\lambda x}\left[-\int \lambda e^{-\lambda x}\left(q_{1}(x)\right)^{-1} q_{2}(x) d x+D\right],
$$

where $D$ is a constant.

Proposition 1.29. Let $X: \Omega \rightarrow(0, \infty)$ be a continuous random variable and let $q_{1}(x)=(1+\beta x)^{-1}$ and $q_{2}(x)=$ $q_{1}(x) e^{-\alpha x}$ for $x>0$. The random variable $X$ has $p d f(63)$, if and only if the function $\xi$ defined in Theorem1 has the form

$$
\xi(x)=\frac{1}{2} e^{-\alpha x}, \quad x>0 .
$$

Corollary 1.29. Let $X: \Omega \rightarrow(0, \infty)$ be a continuous random variable and let $q_{1}(x)$ be as in Proposition 1.29. The random variable $X$ has $p d f(63)$, if and only if there exist functions $q_{2}$ and $\xi$ defined in Theorem 1 satisfying the following differential equation

$$
\frac{\xi^{\prime}(x) q_{1}(x)}{\xi(x) q_{1}(x)-q_{2}(x)}=\alpha, \quad x>0 .
$$

Remark 1.31. The general solution of the differential equation in Corollary 1.29 is

$$
\xi(x)=e^{\alpha x}\left[-\int \alpha e^{-\alpha x}\left(q_{1}(x)\right)^{-1} q_{2}(x) d x+D\right],
$$

where $D$ is a constant.

Proposition 1.30. Let $X: \Omega \rightarrow(0, \infty)$ be a continuous random variable and let $q_{1}(x)=\left[1-\lambda+2 \lambda e^{-\left(\frac{x}{\alpha}\right)^{\beta}}\right]^{-1}\left\{1+(\lambda-1) e^{-\left(\frac{x}{\alpha}\right)^{\beta}}-\lambda e^{-2\left(\frac{x}{\alpha}\right)^{\beta}}\right\}^{1-v}$ and $q_{2}(x)=q_{1}(x) e^{-\left(\frac{x}{\alpha}\right)^{\beta}}$ for $x>0$. The random variable $X$ has $p d f(65)$, if and only if the function $\xi$ defined in Theorem1 has the form

$$
\xi(x)=\frac{1}{2} e^{-\left(\frac{x}{\alpha}\right)^{\beta}}, \quad x>0 .
$$

Corollary 1.30. Let $X: \Omega \rightarrow(0, \infty)$ be a continuous random variable and let $q_{1}(x)$ be as in Proposition 1.30 . The random variable $X$ has $p d f(65)$, if and only if there exist functions $q_{2}$ and $\xi$ defined in Theorem 1 satisfying the following differential equation

$$
\frac{\xi^{\prime}(x) q_{1}(x)}{\xi(x) q_{1}(x)-q_{2}(x)}=\frac{\beta}{\alpha}\left(\frac{x}{\alpha}\right)^{\beta-1}, \quad x>0 .
$$

Remark 1.32. The general solution of the differential equation in Corollary 1.30 is

$$
\xi(x)=e^{\left(\frac{x}{\alpha}\right)^{\beta}}\left[-\int \frac{\beta}{\alpha}\left(\frac{x}{\alpha}\right)^{\beta-1} e^{-\left(\frac{x}{\alpha}\right)^{\beta}}\left(q_{1}(x)\right)^{-1} q_{2}(x) d x+D\right],
$$


where $D$ is a constant.

Proposition 1.31. Let $X: \Omega \rightarrow(0, \infty)$ be a continuous random variable and let $q_{1}(x)=\left(1-e^{1-(1+\alpha x)^{\beta}}\right)^{1-\lambda}$ and $q_{2}(x)=q_{1}(x) e^{1-(1+\alpha x)^{\beta}}$ for $x>0$. The random variable $X$ has $p d f(69)$, if and only if the function $\xi$ defined in Theorem1 has the form

$$
\xi(x)=\frac{1}{2} e^{1-(1+\alpha x)^{\beta}}, \quad x>0 .
$$

Corollary 1.31. Let $X: \Omega \rightarrow(0, \infty)$ be a continuous random variable and let $q_{1}(x)$ be as in Proposition 1.31. The random variable $X$ has $p d f(69)$, if and only if there exist functions $q_{2}$ and $\xi$ defined in Theorem1 satisfying the following differential equation

$$
\frac{\xi^{\prime}(x) q_{1}(x)}{\xi(x) q_{1}(x)-q_{2}(x)}=\alpha \beta(1+\alpha x)^{\beta-1}, \quad x>0 .
$$

Remark 1.33. The general solution of the differential equation in Corollary 1.31 is

$$
\xi(x)=e^{(1+\alpha x)^{\beta}-1}\left[-\int \alpha \beta(1+\alpha x)^{\beta-1} e^{1-(1+\alpha x)^{\beta}}\left(q_{1}(x)\right)^{-1} q_{2}(x) d x+D\right],
$$

where $D$ is a constant.

Proposition 1.32. Let $X: \Omega \rightarrow(0, \infty)$ be a continuous random variable and let $q_{1}(x)=\left(1-e^{-x^{c}}+\alpha e^{-x^{c}}\right)^{\theta+1}$ and $q_{2}(x)=q_{1}(x) e^{1-(1+\alpha x)^{\beta}}$ for $x>0$. The random variable $X$ has $p d f(71)$, if and only if the function $\xi$ defined in Theorem1 has the form

$$
\xi(x)=\frac{1}{2} e^{-x^{c}}, \quad x>0
$$

Corollary 1.32. Let $X: \Omega \rightarrow(0, \infty)$ be a continuous random variable and let $q_{1}(x)$ be as in Proposition 1.32. The random variable $X$ has $p d f(71)$, if and only if there exist functions $q_{2}$ and $\xi$ defined in Theorem 1 satisfying the following differential equation

$$
\frac{\xi^{\prime}(x) q_{1}(x)}{\xi(x) q_{1}(x)-q_{2}(x)}=c x^{c-1}, \quad x>0 .
$$

Remark 1.34. The general solution of the differential equation in Corollary 1.32 is

$$
\xi(x)=e^{x^{c}}\left[-\int c x^{c-1} e^{-x^{c}}\left(q_{1}(x)\right)^{-1} q_{2}(x) d x+D\right],
$$

where $D$ is a constant.

Proposition 1.33. Let $X: \Omega \rightarrow \mathbb{R}$ be a continuous random variable and let $q_{1}(x) \equiv 1$ and $q_{2}(x)=\exp \left\{-c[-\ln (\bar{G}(x ; \eta))]^{d}-a[-\ln (\bar{G}(x ; \eta))]^{b}\right\}$ for $x \in \mathbb{R}$. The random variable $X$ has $p d f(75)$, if and only if the function $\xi$ defined in Theorem 1 has the form

$$
\xi(x)=\frac{1}{2}\left\{1-\exp \left\{-c[-\ln (\bar{G}(x ; \eta))]^{d}-a[-\ln (\bar{G}(x ; \eta))]^{b}\right\}\right\}, x \in \mathbb{R} .
$$

Corollary 1.33. Let $X: \Omega \rightarrow \mathbb{R}$ be a continuous random variable and let $q_{1}(x)$ be as in Proposition 1.33. The random variable $X$ has $p d f(75)$, if and only if there exist functions $q_{2}$ and $\xi$ defined in Theorem1 satisfying the following differential equation

$$
\begin{aligned}
& \frac{\xi^{\prime}(x) q_{1}(x)}{\xi(x) q_{1}(x)-q_{2}(x)} \\
& \quad \frac{\frac{g(x ; \eta)}{\bar{G}(x ; \eta)}\left\{c d[-\ln (\bar{G}(x ; \eta))]^{d-1}+a b[-\ln (\bar{G}(x ; \eta))]^{b-1}\right\} \times}{\exp \left\{-c[-\ln (\bar{G}(x ; \eta))]^{d}-a[-\ln (\bar{G}(x ; \eta))]^{b}\right\}} \\
& =\frac{\exp \left\{-c[-\ln (\bar{G}(x ; \eta))]^{d}-a[-\ln (\bar{G}(x ; \eta))]^{b}\right\}}{1-\operatorname{ex}}, x \in \mathbb{R} .
\end{aligned}
$$

Remark 1.35. The general solution of the differential equation in Corollary 1.33 is

$$
\begin{aligned}
& \xi(x)=\left\{1-\exp \left\{-c[-\ln (\bar{G}(x ; \eta))]^{d}-a[-\ln (\bar{G}(x ; \eta))]^{b}\right\}\right\}^{-1} \times \\
& {\left[\begin{array}{c}
-\int \frac{g(x ; \eta)}{\bar{G}(x ; \eta)}\left\{c d[-\ln (\bar{G}(x ; \eta))]^{d-1}+a b[-\ln (\bar{G}(x ; \eta))]^{b-1}\right\} \times \\
\exp \left\{-c[-\ln (\bar{G}(x ; \eta))]^{d}-a[-\ln (\bar{G}(x ; \eta))]^{b}\right\} q_{2}(x) d x+D
\end{array}\right],}
\end{aligned}
$$


where $D$ is a constant.

Proposition 1.34. Let $X: \Omega \rightarrow(0, \infty)$ be a continuous random variable and let $q_{1}(x)=\left(1-e^{-\lambda \alpha_{1} x}\right)^{-1}\left(1-e^{-\lambda \alpha_{2} x}\right)^{-1}$ and $q_{2}(x)=q_{1}(x) e^{-\lambda x}$ for $x>0$. The random variable $X$ has $p d f(77)$, if and only if the function $\xi$ defined in Theorem1 has the form

$$
\xi(x)=\frac{1}{2} e^{-\lambda x}, x>0 .
$$

Corollary 1.34. Let $X: \Omega \rightarrow(0, \infty)$ be a continuous random variable and let $q_{1}(x)$ be as in Proposition 1.34. The random variable $X$ has $p d f(77)$, if and only if there exist functions $q_{2}$ and $\xi$ defined in Theorem 1 satisfying the following differential equation

$$
\frac{\xi^{\prime}(x) q_{1}(x)}{\xi(x) q_{1}(x)-q_{2}(x)}=\lambda, \quad x>0 .
$$

Remark 1.36. The general solution of the differential equation in Corollary 1.34 is

$$
\xi(x)=e^{\lambda x}\left[-\int \lambda e^{-\lambda x}\left(q_{1}(x)\right)^{-1} q_{2}(x) d x+D,\right]
$$

where $D$ is a constant.

Proposition 1.35. Let $X: \Omega \rightarrow(0, \infty)$ be a continuous random variable and let $q_{1}(x)=\left(1-e^{-\lambda(\alpha x)^{\beta}}\right)^{-v}$ and $q_{2}(x)=$ $q_{1}(x) e^{-\lambda x^{\beta}}$ for $x>0$. The random variable $X$ has $p d f(78)$, if and only if the function $\xi$ defined in Theorem1 has the form

$$
\xi(x)=\frac{1}{2} e^{-\lambda x^{\beta}}, x>0 .
$$

Corollary 1.35. Let $X: \Omega \rightarrow(0, \infty)$ be a continuous random variable and let $q_{1}(x)$ be as in Proposition 1.35. The random variable $X$ has $p d f(78)$, if and only if there exist functions $q_{2}$ and $\xi$ defined in Theorem 1 satisfying the following differential equation

$$
\frac{\xi^{\prime}(x) q_{1}(x)}{\xi(x) q_{1}(x)-q_{2}(x)}=\lambda \beta x^{\beta-1}, x>0 .
$$

Remark 1.37. The general solution of the differential equation in Corollary 1.35 is

$$
\xi(x)=e^{\lambda x^{\beta}}\left[-\int \lambda \beta e^{-\lambda x^{\beta}}\left(q_{1}(x)\right)^{-1} q_{2}(x) d x+D,\right]
$$

where $D$ is a constant.

Proposition 1.36. Let $X: \Omega \rightarrow(0, \infty)$ be a continuous random variable and let $q_{1}(x)=e^{(x / \theta)^{\lambda}}$ and $q_{2}(x)=$ $q_{1}(x)\left(1-e^{-(x / \theta)^{\lambda}}\right)^{2}$ for $x>0$. The random variable $X$ has $p d f(80)$, if and only if the function $\xi$ defined in Theorem1 has the form

$$
\xi(x)=\frac{1}{2}\left\{1+\left(1-e^{-(x / \theta)^{\lambda}}\right)^{2}\right\}, x>0 .
$$

Corollary 1.36. Let $X: \Omega \rightarrow(0, \infty)$ be a continuous random variable and let $q_{1}(x)$ be as in Proposition 1.36. The random variable $X$ has $p d f(80)$, if and only if there exist functions $q_{2}$ and $\xi$ defined in Theorem1 satisfying the following differential equation

$$
\frac{\xi^{\prime}(x) q_{1}(x)}{\xi(x) q_{1}(x)-q_{2}(x)}=\frac{\frac{2 \lambda}{\theta}\left(\frac{x}{\theta}\right)^{\lambda-1} e^{-(x / \theta)^{\lambda}}\left(1-e^{-(x / \theta)^{\lambda}}\right)}{1-\left(1-e^{-(x / \theta)^{\lambda}}\right)^{2}}, x>0 .
$$

Remark 1.38. The general solution of the differential equation in Corollary 1.36 is

$$
\begin{aligned}
\xi(x) & =\left\{1-\left(1-e^{-(x / \theta)^{\lambda}}\right)^{2}\right\}^{-1} \times \\
& {\left[-\int \frac{2 \lambda}{\theta}\left(\frac{x}{\theta}\right)^{\lambda-1} e^{-(x / \theta)^{\lambda}}\left(1-e^{-(x / \theta)^{\lambda}}\right)\left(q_{1}(x)\right)^{-1} q_{2}(x) d x+D,\right] }
\end{aligned}
$$

where $D$ is a constant.

\subsection{Characterization in Terms of Hazard Function}

The hazard function, $h_{F}$, of a twice differentiable distribution function, $F$, satisfies the following first order differential equation

$$
\frac{f^{\prime}(x)}{f(x)}=\frac{h_{F}^{\prime}(x)}{h_{F}(x)}-h_{F}(x)
$$


It should be mentioned that for many univariate continuous distributions, the above equation is the only differential equation available in terms of the hazard function. In this subsection we present non-trivial characterizations of EGLFR (for $\alpha=1$ ), McG (for $a=b=c=1$ ), Go-G, EGGP (for $b=1$ ), BIR (for $a=1$ ), BWP (for $a=1$ ), EGG (for $\alpha=1$ ), NEPL (for $\alpha=1$ ), EGGG, ETQL (for $\lambda=0, \beta=1$ ), ERK (for $\lambda=1$ ), AEE, NEE, ETW (for $v=1$ ), ENHE (for $\lambda=1$ ), GW (for $\theta=1$ ), GAW-G (for $b=0$ or $d=0$ ), GB distributions in terms of the hazard function, which are not of the above trivial form.

Proposition 2.1. Let $X: \Omega \rightarrow(0, \infty)$ be a continuous random variable. The random variable $X$ has $p d f(2)$ (for $\beta<0$ and $\alpha=1$ ) if and only if its hazard function $h_{F}(x)$ satisfies the following differential equation

$$
h_{F}^{\prime}(x)-\frac{b}{a+b x} h_{F}(x)=\frac{\beta(a+b x)^{2}}{\left[1-\beta\left(a x+\frac{b}{2} x^{2}\right)\right]^{2}}, \quad x>0 .
$$

Proof. If $X$ has $p d f$ (2) for $\beta<0$ and $\alpha=1$, then clearly the above differential equation holds. If the differential equation holds, then

$$
\begin{aligned}
\frac{d}{d x}\left\{(a+b x)^{-1} h_{F}(x)\right\} & =\frac{\beta(a+b x)}{\left[1-\beta\left(a x+\frac{b}{2} x^{2}\right)\right]^{2}} \\
& =\frac{d}{d x}\left\{\left[1-\beta\left(a x+\frac{b}{2} x^{2}\right)\right]^{-1}\right\},
\end{aligned}
$$

from which we arrive at the hazard function corresponding to the $p d f(2)$.

Remark 2.1. Similar Propositions can be stated for the cases $I I$ and $I I I$.

A Proposition similar to that of Proposition 2.1 will be stated (without proof) for each one of the distributions listed in subsection 2.1.

Proposition 2.2. Let $X: \Omega \rightarrow(0, \infty)$ be a continuous random variable. The random variable $X$ has $p d f$ (4) (for $a=b=c=1)$ if and only if its hazard function $h_{F}(x)$ satisfies the following differential equation

$$
h_{F}^{\prime}(x)-\gamma h_{F}(x)=0, \quad x>0 .
$$

Proposition 2.3. Let $X: \Omega \rightarrow \mathbb{R}$ be a continuous random variable. The random variable $X$ has $p d f(12)$ if and only if its hazard function $h_{F}(x)$ satisfies the following differential equation

$$
h_{F}^{\prime}(x)-\frac{g^{\prime}(x ; \eta)}{g(x ; \eta)} h_{F}(x)=\theta(\gamma+1)(g(x ; \eta))^{2}(\bar{G}(x ; \eta))^{-\gamma-2}, \quad x \in \mathbb{R} .
$$

Proposition 2.4. Let $X: \Omega \rightarrow(0, \infty)$ be a continuous random variable. The random variable $X$ has $p d f(20)$ if and only if its hazard function $h_{F}(x)$ satisfies the following differential equation

$$
\begin{gathered}
h_{F}^{\prime}(x)-\frac{g^{\prime}(x ; \zeta)}{g(x ; \zeta)} h_{F}(x)=2 \lambda[g(x ; \zeta)]^{2} \times \\
\left\{\frac{\lambda-1}{1-[G(x ; \zeta)]^{2 \lambda}}+\frac{2 \lambda[G(x ; \zeta)]^{3 \lambda-1}}{\left[1-[G(x ; \zeta)]^{2 \lambda}\right]^{2}}\right\}, \quad x>0 .
\end{gathered}
$$

Proposition 2.5. Let $X: \Omega \rightarrow \mathbb{R}$ be a continuous random variable. The random variable $X$ has $p d f(28)$, for $b=1$, if and only if its hazard function $h_{F}(x)$ satisfies the following differential equation

$$
\begin{aligned}
& h_{F}^{\prime}(x)-\frac{g^{\prime}(x ; \psi)}{g(x ; \psi)} h_{F}(x)=a \lambda[g(x ; \psi)]^{2}[\bar{G}(x ; \psi)]^{a-1} \times \\
& \frac{\left\{\left[(a-1)+a \lambda[\bar{G}(x ; \psi)]^{a-1}\right] \exp \left\{-\lambda[\bar{G}(x ; \psi)]^{a}\right\}-(a-1)\right\}}{\left(1-\exp \left\{-\lambda[\bar{G}(x ; \psi)]^{a}\right\}\right)^{2}},
\end{aligned}
$$

$x \in \mathbb{R}$.

Proposition 2.6. Let $X: \Omega \rightarrow(0, \infty)$ be a continuous random variable. The random variable $X$ has $p d f(30)$, for $a=1$, if and only if its hazard function $h_{F}(x)$ satisfies the following differential equation

$$
h_{F}^{\prime}(x)-2 \theta x^{-3} h_{F}(x)=\frac{2 \theta b \exp \left(-\theta x^{-2}\right)\left[\left(2 \theta x^{-2}+3\right) \exp \left(-\theta x^{-2}\right)-3\right]}{x^{4}\left[1-\exp \left(-\theta x^{-2}\right)\right]^{2}}, x>0 .
$$


Proposition 2.7. Let $X: \Omega \rightarrow(0, \infty)$ be a continuous random variable. The random variable $X$ has $p d f(34)$, for $a=1$, if and only if its hazard function $h_{F}(x)$ satisfies the following differential equation

$$
h_{F}^{\prime}(x)-(\alpha-1) x^{-1} h_{F}(x)=b \alpha \beta \lambda x^{\alpha-1} \frac{d}{d x}\left\{\frac{e^{-\beta x^{\alpha}} e^{\lambda e^{-\beta x^{\alpha}}}}{e^{\lambda e^{-\beta x^{\alpha}}}-1}\right\}, x>0,
$$

or

$$
h_{F}^{\prime}(x)+\alpha \beta x^{\beta-1} h_{F}(x)=b \alpha \beta \lambda e^{-\beta x^{\alpha}} \frac{d}{d x}\left\{\frac{x^{\alpha-1} e^{\lambda e^{-\beta x^{\alpha}}}}{e^{\lambda e^{-\beta x^{\alpha}}}-1}\right\}, x>0 .
$$

Proposition 2.8. Let $X: \Omega \rightarrow \mathbb{R}$ be a continuous random variable. The random variable $X$ has $p d f(40)$, for $\alpha=1$, if and only if its hazard function $h_{F}(x)$ satisfies the following differential equation

$$
h_{F}^{\prime}(x)-\frac{g^{\prime}(x ; \eta)}{g(x ; \eta)} h_{F}(x)=\alpha \theta(\gamma+1)(g(x ; \eta))^{2}(\bar{G}(x ; \eta))^{-\gamma-2}, x \in \mathbb{R} .
$$

Proposition 2.9. Let $X: \Omega \rightarrow(0, \infty)$ be a continuous random variable. The random variable $X$ has $p d f(42)$, for $\alpha=1$, if and only if its hazard function $h_{F}(x)$ satisfies the following differential equation

$$
h_{F}^{\prime}(x)-(\beta-1) x^{-1} h_{F}(x)=\frac{\beta^{2} \lambda^{2} x^{2(\beta-1)}}{\left(1+\lambda+\lambda x^{\beta}\right)^{2}}, x>0 .
$$

Proposition 2.10. Let $X: \Omega \rightarrow(0, \infty)$ be a continuous random variable. The random variable $X$ has $p d f(48)$, if and only if its hazard function $h_{F}(x)$ satisfies the following differential equation

$$
h_{F}^{\prime}(x)+\frac{\tau}{\alpha}\left(\frac{x}{\alpha}\right)^{\tau-1} h_{F}(x)=\frac{\lambda \tau \exp \left(-\left(\frac{x}{\alpha}\right)^{\tau}\right)}{\alpha \Gamma(k)} \frac{d}{d x}\left\{\frac{\left(\frac{x}{\alpha}\right)^{\tau k-1}\left(1-\gamma_{1}\left[k,\left(\frac{x}{\alpha}\right)^{\tau}\right]\right)^{-1}}{\left(1-p\left\{1-\gamma_{1}\left[k,\left(\frac{x}{\alpha}\right)^{\tau}\right]\right\}\right)}\right\}, x>0 .
$$

Remark 2.2. For $k=1$, the above differential equation has the following simpler form

$$
h_{F}^{\prime}(x)-\frac{\tau-1}{x} h_{F}(x)=-\frac{\lambda \tau^{2} p\left(\frac{x}{\alpha}\right)^{2(\tau-1)} \exp \left(-\left(\frac{x}{\alpha}\right)^{\tau}\right)}{\alpha^{2}\left(1-p \exp \left(-\left(\frac{x}{\alpha}\right)^{\tau}\right)\right)^{2}}, x>0 .
$$

Proposition 2.11. Let $X: \Omega \rightarrow(0, \infty)$ be a continuous random variable. The random variable $X$, has $p d f(50)$, for $\lambda=0, \beta=1$, if and only if its hazard function $h_{F}(x)$ satisfies the following differential equation

$$
h_{F}^{\prime}(x)-(\mu+x)^{-1} h_{F}(x)=-\frac{\alpha \theta^{3}(\mu+x)}{(\mu \theta+1+\theta x)^{2}}, x>0 .
$$

Proposition 2.12. Let $X: \Omega \rightarrow(0,1)$ be a continuous random variable. The random variable $X$, has $p d f(53)$, for $\lambda=1$, if and only if its hazard function $h_{F}(x)$ satisfies the following differential equation

$$
h_{F}^{\prime}(x)-(\beta-1) x^{-1} h_{F}(x)=\beta(\beta+1) x^{\beta-1}(1-x)^{-(\beta+2)}, x \in(0,1) .
$$

Proposition 2.13. Let $X: \Omega \rightarrow(0, \infty)$ be a continuous random variable. The random variable $X$, has $p d f(57)$ if and only if its hazard function $h_{F}(x)$ satisfies the following differential equation

$$
h_{F}^{\prime}(x)-(1+\beta x)^{-1} h_{F}(x)=-\alpha^{3} \beta(1+\beta x)(\alpha+\beta+\alpha \beta x)^{-2}, \quad x>0 .
$$

Proposition 2.14. Let $X: \Omega \rightarrow(0, \infty)$ be a continuous random variable. The random variable $X$, has $p d f(63)$, if and only if its hazard function $h_{F}(x)$ satisfies the following differential equation

$$
h_{F}^{\prime}(x)-\frac{1}{1+\lambda x} h_{F}(x)=-\frac{\alpha^{3} \beta(1+\beta x)}{(\alpha+\beta+\alpha \beta x)^{2}}, \quad x>0 .
$$

Proposition 2.15. Let $X: \Omega \rightarrow(0, \infty)$ be a continuous random variable. The random variable $X$, has $p d f(65)$, for $v=1$, if and only if its hazard function $h_{F}(x)$ satisfies the following differential equation

$$
h_{F}^{\prime}(x)-(\beta-1) x^{-1} h_{F}(x)=\frac{v \beta}{\alpha}\left(\frac{x}{\beta}\right)^{\beta-1} \frac{d}{d x}\left\{\frac{1-\lambda+2 \lambda e^{-\left(\frac{x}{\alpha}\right)^{\beta}}}{\lambda-1-\lambda e^{-\left(\frac{x}{\alpha}\right)^{\beta}}}\right\}, \quad x>0 .
$$


Proposition 2.16. Let $X: \Omega \rightarrow(0, \infty)$ be a continuous random variable. The random variable $X$, has $p d f(69)$, for $\lambda=1$, if and only if its hazard function $h_{F}(x)$ satisfies the following differential equation

$$
h_{F}^{\prime}(x)-\frac{\alpha(\beta-1)}{1+\alpha x} h_{F}(x)=0, \quad x>0 .
$$

Proposition 2.17. Let $X: \Omega \rightarrow(0, \infty)$ be a continuous random variable. The random variable $X$, has $p d f(71)$, for $\theta=1$, if and only if its hazard function $h_{F}(x)$ satisfies the following differential equation

$$
h_{F}^{\prime}(x)-(c-1) x^{-1} h_{F}(x)=(1-\alpha) c^{2} x^{2(c-1)} e^{-x^{c}}, \quad x>0 .
$$

Proposition 2.18. Let $X: \Omega \rightarrow \mathbb{R}$ be a continuous random variable. The random variable $X$, has $p d f(73)$, for $d=0$, if and only if its hazard function $h_{F}(x)$ satisfies the following differential equation

$$
\begin{aligned}
& h_{F}^{\prime}(x)-\frac{g^{\prime}(x ; \eta)}{g(x ; \eta)} h_{F}(x) \\
& =\frac{a b(g(x ; \eta))^{2}[-\ln (\bar{G}(x ; \eta))]^{b-2}\{b-1-\ln (\bar{G}(x ; \eta))\}}{(\bar{G}(x ; \eta))^{2}}, x \in \mathbb{R} .
\end{aligned}
$$

Proposition 2.19. Let $X: \Omega \rightarrow(0, \infty)$ be a continuous random variable. The random variable $X$ has $p d f(80)$ if and only if its hazard function $h_{F}(x)$ satisfies the following differential equation

$$
h_{F}^{\prime}(x)-(\lambda-1) x^{-1} h_{F}(x)=\frac{6 \lambda^{2}}{\theta^{2}}\left(\frac{x}{\theta}\right)^{2(\lambda-1)} e^{-(x / \theta)^{\lambda}}, \quad x>0 .
$$

\subsection{Characterization in Terms of the Reverse (or reversed) Hazard Function}

The reverse hazard function, $r_{F}$, of a twice differentiable distribution function, $F$, is defined as

$$
r_{F}(x)=\frac{f(x)}{F(x)}, x \in \text { support of } F \text {. }
$$

In this subsection we present characterizations of EGLFR, McG (for $b=1$ ), Kw-TEMW (for $b=1, \lambda=0$ ), ALLGE , Po-G, EGGP (for $a=1$ ), BIR (for $b=1$ ), BWP (for $b=1$ ), GMW (for $a=1$ ), TW (for $\lambda=0$ ), PWR (for $\mu=0, \sigma=1$ ), OLLSN (for $\mu=0, \sigma=1$ ), EGGG (for $\lambda=1$ ), ETQL (for $\lambda=0$ ), ERK, ETW, ENHD, GWW (for $\alpha=1$ ) distributions (without proofs) in terms of the reverse hazard function.

Proposition 3.1. Let $X: \Omega \rightarrow(0, \infty)$ be a continuous random variable. The random variable $X$ has $p d f(2)$ (for $\beta<0)$ if and only if its reverse hazard function $r_{F}(x)$ satisfies the following differential equation

$$
r_{F}^{\prime}(x)-\frac{b}{a+b x} r_{F}(x)=\alpha(a+b x) \frac{d}{d x}\left\{\frac{\left(1-\beta\left(a x+\frac{b}{2} x^{2}\right)\right)^{\frac{1}{\beta}-1}}{1-\left(1-\beta\left(a x+\frac{b}{2} x^{2}\right)\right)^{1 / \beta}}\right\}, \quad x>0 .
$$

Proposition 3.2. Let $X: \Omega \rightarrow(0, \infty)$ be a continuous random variable. The random variable $X$ has $p d f(4)$ (for $b=1)$ if and only if its reverse hazard function $r_{F}(x)$ satisfies the following differential equation

$$
r_{F}^{\prime}(x)-\gamma r_{F}(x)=-\frac{a c \theta^{2} e^{\gamma x} \exp \left(\frac{\theta}{\gamma}\left(e^{\gamma x}-1\right)\right)}{\left(\exp \left(\frac{\theta}{\gamma}\left(e^{\gamma x}-1\right)\right)-1\right)^{2}}, x>0 .
$$

Proposition 3.3. Let $X: \Omega \rightarrow(0, \infty)$ be a continuous random variable. The random variable $X$ has $p d f(6)$ (for $b=1$, $\lambda=0)$ if and only if its reverse hazard function $r_{F}(x)$ satisfies the following differential equation

$$
r_{F}^{\prime}(x)+\left(\theta+\gamma \beta x^{\beta-1}\right) r_{F}(x)=\alpha a e^{-\left(\theta x+\gamma x^{\beta}\right)} \frac{d}{d x}\left\{\frac{\theta+\gamma \beta x^{\beta-1}}{1-e^{-\left(\theta x+\gamma x^{\beta}\right)}}\right\}, x>0 .
$$

Proposition 3.4. Let $X: \Omega \rightarrow(0, \infty)$ be a continuous random variable. The random variable $X$ has $p d f(18)$ if and only if its reverse hazard function $r_{F}(x)$ satisfies the following differential equation

$$
r_{F}^{\prime}(x)+\eta r_{F}(x)=\eta \theta \delta^{\theta} e^{-\eta x} \frac{d}{d x}\left\{\frac{1}{\left(1-e^{-\eta x}\right)\left[\delta^{\theta}+\left(1-e^{-\eta x}\right)^{\theta}\right]}\right\}, x>0 .
$$


Proposition 3.5. Let $X: \Omega \rightarrow(0, \infty)$ be a continuous random variable. The random variable $X$ has $p d f(20)$ if and only if its reverse hazard function $r_{F}(x)$ satisfies the following differential equation

$$
r_{F}^{\prime}(x)-\frac{g^{\prime}(x ; \zeta)}{g(x ; \zeta)} r_{F}(x)=-\lambda[g(x ; \zeta)]^{2}\left\{\frac{1-\lambda[G(x ; \zeta)]^{\lambda-2}+[G(x ; \zeta)]^{\lambda}}{\left[1+[G(x ; \zeta)]^{\lambda}\right]^{2}}\right\}, x>0 .
$$

Proposition 3.6. Let $X: \Omega \rightarrow \mathbb{R}$ be a continuous random variable. The random variable $X$ has $p d f(24)$ if and only if its reverse hazard function $r_{F}(x)$ satisfies the following differential equation

$$
r_{F}^{\prime}(x)-\frac{g^{\prime}(x ; \eta)}{g(x ; \eta)} r_{F}(x)=-\frac{\theta^{2}[g(x ; \eta)]^{2} \exp [\theta G(x ; \eta)]}{\{\exp [\theta G(x ; \eta)]-1\}}, x \in \mathbb{R} .
$$

Proposition 3.7. Let $X: \Omega \rightarrow \mathbb{R}$ be a continuous random variable. The random variable $X$ has $p d f(28)$ if and only if its reverse hazard function $r_{F}(x)$ satisfies the following differential equation

$$
\begin{aligned}
r_{F}^{\prime}(x)-\frac{g^{\prime}(x ; \psi)}{g(x ; \psi)} r_{F}(x) & =b(b-1) \lambda[g(x ; \psi)]^{2}[G(x ; \psi)]^{b-1} \times \\
& \frac{\left\{\left[(b-1)-b \lambda[G(x ; \psi)]^{b-1}\right] \exp \left\{\lambda[G(x ; \psi)]^{b}\right\}-(b-1)\right\}}{\left(\exp \left\{\lambda[G(x ; \psi)]^{b}\right\}-1\right)^{2}},
\end{aligned}
$$

$x \in \mathbb{R}$. Proposition 3.8. Let $X: \Omega \rightarrow(0, \infty)$ be a continuous random variable. The random variable $X$ has $p d f(30)$, for $b=1$, if and only if its reverse hazard function $r_{F}(x)$ satisfies the following differential equation

$$
h_{F}^{\prime}(x)+3 x^{-1} h_{F}(x)=0, \quad x>0 .
$$

Proposition 3.9. Let $X: \Omega \rightarrow(0, \infty)$ be a continuous random variable. The random variable $X$ has $p d f(34)$,for $b=1$, if and only if its reverse hazard function $r_{F}(x)$ satisfies the following differential equation

$$
r_{F}^{\prime}(x)+\alpha \beta x^{\beta-1} r_{F}(x)=a \alpha \beta \lambda e^{-\beta x^{\alpha}} \frac{d}{d x}\left\{\frac{x^{\alpha-1} e^{\lambda e^{-\beta x^{\alpha}}}}{e^{\lambda}-e^{\lambda e^{-\beta x^{\alpha}}}}\right\}, \quad x>0 .
$$

Proposition 3.10. Let $X: \Omega \rightarrow(0, \infty)$ be a continuous random variable. The random variable $X$ has $p d f(36)$,for $a=1$, if and only if its reverse hazard function $r_{F}(x)$ satisfies the following differential equation

$$
r_{F}^{\prime}(x)-\lambda r_{F}(x)=\beta x^{\beta-2}(\beta-1+\lambda x) e^{\lambda x}, \quad x>0 .
$$

Proposition 3.11. Let $X: \Omega \rightarrow(0, \infty)$ be a continuous random variable. The random variable $X$ has $p d f(38)$,for $\lambda=0$, if and only if its reverse hazard function $r_{F}(x)$ satisfies the following differential equation

$$
r_{F}^{\prime}(x)+(\theta+1) x^{-1} r_{F}(x)=\alpha^{2} \beta \theta^{2} x^{-2(\theta+1)}\left(1+\alpha x^{-\theta}\right)^{-2}, \quad x>0 .
$$

Proposition 3.12. Let $X: \Omega \rightarrow \mathbb{R}$ be a continuous random variable. The random variable $X$ has $p d f(44)$ if and only if its reverse hazard function $r_{F}(x)$ satisfies the following differential equation

$$
r_{F}^{\prime}(x)-r_{F}(x)=\alpha e^{x} \frac{d}{d x}\left\{\frac{e^{\alpha e^{-x}-e^{x}}}{e^{\alpha}-e^{\alpha e^{-x}}}\right\}, \quad x \in \mathbb{R} .
$$

Proposition 3.13. Let $X: \Omega \rightarrow \mathbb{R}$ be a continuous random variable. The random variable $X$ has $p d f(46)$ if and only if its reverse hazard function $r_{F}(x)$ satisfies the following differential equation

$$
r_{F}^{\prime}(x)-\frac{\lambda \phi(x)}{\Phi(\lambda x)} r_{F}(x)=2 \alpha \Phi(\lambda x) \frac{d}{d x}\left\{\frac{\phi(x)\left[\bar{\Phi}_{S N}(x ; \lambda)\right]^{\alpha-1}}{\Phi_{S N}(x ; \lambda)\left\{\left[\Phi_{S N}(x ; \lambda)\right]^{\alpha}+\left[\bar{\Phi}_{S N}(x ; \lambda)\right]^{\alpha}\right\}}\right\}, \quad x \in \mathbb{R} .
$$

Proposition 3.14. Let $X: \Omega \rightarrow(0, \infty)$ be a continuous random variable. The random variable $X$ has $p d f(48)$,for $\lambda=1$, if and only if its reverse hazard function $r_{F}(x)$ satisfies the following differential equation

$$
r_{F}^{\prime}(x)-\frac{\tau-1}{x} r_{F}(x)=\frac{\tau(1-p) \exp \left(-\left(\frac{x}{\alpha}\right)^{\tau}\right)}{\alpha \Gamma(k)} \frac{d}{d x}\left\{\frac{\left(\frac{x}{\alpha}\right)^{\tau k-1}\left(\gamma_{1}\left[k,\left(\frac{x}{\alpha}\right)^{\tau}\right]\right)^{-1}}{1-p\left\{1-\gamma_{1}\left[k,\left(\frac{x}{\alpha}\right)^{\tau}\right]\right\}}\right\}, x>0 .
$$


Proposition 3.15. Let $X: \Omega \rightarrow(0, \infty)$ be a continuous random variable. The random variable $X$ has $p d f(50)$, for $\lambda=0$, if and only if its reverse hazard function $r_{F}(x)$ satisfies the following differential equation

$$
r_{F}^{\prime}(x)+\theta r_{F}(x)=\frac{\alpha \beta \theta^{2}}{\mu \theta+1} e^{-\theta x} \frac{d}{d x}\left\{\frac{(\mu+x)\left(\frac{\mu \theta+1+\theta x}{\mu \theta+1} e^{-\theta x}\right)^{\alpha-1}}{1-\left(\frac{\mu \theta+1+\theta x}{\mu \theta+1}\right)^{\alpha} e^{-\alpha \theta x}}\right\}, x>0 .
$$

Proposition 3.16. Let $X: \Omega \rightarrow(0,1)$ be a continuous random variable. The random variable $X$ has $p d f(53)$, if and only if its reverse hazard function $r_{F}(x)$ satisfies the following differential equation

$$
r_{F}^{\prime}(x)+\frac{\beta x^{\beta-1}}{(1-x)^{\beta+1}} r_{F}(x)=\lambda \beta \exp \left[-\left(\frac{x}{1-x}\right)^{\beta}\right] \frac{d}{d x}\left\{\frac{x^{\beta-1}(1-x)^{-(\beta+1)}}{1-\exp \left[-\left(\frac{x}{1-x}\right)^{\beta}\right]}\right\}, x>0 .
$$

Proposition 3.17. Let $X: \Omega \rightarrow(0, \infty)$ be a continuous random variable. The random variable $X$ has $p d f(65)$, if and only if its reverse hazard function $r_{F}(x)$ satisfies the following differential equation

$$
r_{F}^{\prime}(x)+\frac{\beta}{\alpha}\left(\frac{x}{\beta}\right)^{\beta-1} r_{F}(x)=\frac{v \beta}{\alpha} \frac{d}{d x}\left\{\frac{\left(\frac{x}{\alpha}\right)^{\beta-1}\left[1-\lambda+2 \lambda e^{-\left(\frac{x}{\alpha}\right)^{\beta}}\right]}{1+(\lambda-1) e^{-\left(\frac{x}{\alpha}\right)^{\beta}}-\lambda e^{-2\left(\frac{x}{\alpha}\right)^{\beta}}}\right\}, \quad x>0 .
$$

Proposition 3.18. Let $X: \Omega \rightarrow(0, \infty)$ be a continuous random variable. The random variable $X$ has $p d f(69)$, if and only if its reverse hazard function $r_{F}(x)$ satisfies the following differential equation

$$
r_{F}^{\prime}(x)+\alpha \beta(1+\alpha x)^{\beta-1} r_{F}(x)=\lambda \alpha \beta e^{1-(1+\alpha x)^{\beta}} \frac{d}{d x}\left\{\frac{(1+\alpha x)^{\beta-1}}{1-e^{1-(1+\alpha x)^{\beta}}}\right\}, \quad x>0 .
$$

Proposition 3.19. Let $X: \Omega \rightarrow(0, \infty)$ be a continuous random variable. The random variable $X$ has $p d f(78)$, for $\alpha=1$, if and only if its reverse hazard function $r_{F}(x)$ satisfies the following differential equation

$$
\begin{aligned}
& r_{F}^{\prime}(x)+\lambda \beta x^{\beta-1} r_{F}(x) \\
& =\frac{\lambda \beta(v+1) x^{\beta-2} e^{-\lambda x^{\beta}}\left\{(\beta-1)\left(1-e^{-\lambda x^{\beta}}\right)-\lambda \beta x^{\beta} e^{-\lambda x^{\beta}}\right\}}{\left(1-e^{-\lambda x^{\beta}}\right)^{2}}, \quad x>0 .
\end{aligned}
$$

\subsection{Characterization Based on the Conditional Expectation of Certain Function of the Random Variable}

In this subsection we employ a single function $\psi$ (or $\psi_{1}$ ) of $X$ and characterize the distribution of $X$ in terms of the truncated moment of $\psi(X)$ (or $\psi_{1}(X)$ ). The following propositions have already appeared in Hamedani's previous work (2013), so we will just state them here which can be used to characterize some of the distributions listed in Section 1.

Proposition H1. Let $X: \Omega \rightarrow(e, f)$ be a continuous random variable with $c d f F$. Let $\psi(x)$ be a differentiable function on $(e, f)$ with $\lim _{x \rightarrow e^{+}} \psi(x)=1$. Then for $\delta \neq 1$,

$$
E[\psi(X) \mid X \geq x]=\delta \psi(x), \quad x \in(e, f)
$$

if and only if

$$
\psi(x)=(1-F(x))^{\frac{1}{\delta}-1}, \quad x \in(e, f)
$$

Proposition H2. Let $X: \Omega \rightarrow(e, f)$ be a continuous random variable with $c d f F$. Let $\psi_{1}(x)$ be a differentiable function on $(e, f)$ with $\lim _{x \rightarrow f^{-}} \psi_{1}(x)=1$. Then for $\delta_{1} \neq 1$,

$$
E\left[\psi_{1}(X) \mid X \leq x\right]=\delta_{1} \psi_{1}(x), \quad x \in(e, f)
$$

implies

$$
\psi_{1}(x)=(F(x))^{\frac{1}{\delta_{1}}-1} . \quad x \in(e, f)
$$

Remarks 4.1. (A) For $(e, f)=(0, \infty), \alpha=1, \psi(x)=\left(1-\beta\left(a x+\frac{b}{2} x^{2}\right)\right)$ and $\delta=\frac{1}{1+\beta}$, Proposition H1 provides a characterization of EGLFR for $\beta<0$. (B) For $(e, f)=(0, \infty), \psi_{1}(x)=1-\left(1-\beta\left(a x+\frac{b}{2} x^{2}\right)\right)^{1 / \beta}$ and $\delta_{1}=\frac{\alpha}{\alpha+1}$ 
, Proposition H2 provides a characterization of EGLFR for $\beta<0$. Statements similar to $(A)$ and $(B)$ can be made for Cases $I I$ and III as well. (C) For $(e, f)=(0, \infty), b=c=1, \psi(x)=\exp \left(-\frac{\theta}{\gamma}\left(e^{\gamma x}-1\right)\right)$ and $\delta=\frac{1}{2}$, Proposition H1 provides a characterization of McG distribution. (D) For $(e, f)=(0, \infty), \lambda=0, \psi(x)=1-\left[1-e^{-\left(\theta x+\gamma x^{\beta}\right)}\right]^{\alpha a}$ and $\delta=\frac{b}{b+1}$, Proposition H1 provides a characterization of Kw-TEMW distribution. $(E)$ For $(e, f)=(0, \infty), b=1$ ,$\psi_{1}(x)=1-\exp \left(-\frac{\theta}{\gamma}\left(e^{\gamma x}-1\right)\right)$ and $\delta_{1}=\frac{c}{c+1}$, Proposition $\mathrm{H} 2$ provides a characterization of McG distribution. $(F)$ For $(e, f)=(0, \infty), b=1, \lambda=0, \psi_{1}(x)=1-e^{-\left(\theta x+\gamma x^{\beta}\right)}$ and $\delta=\frac{\alpha a}{1+\alpha a}$, Proposition H2 provides a characterization of Kw-TEMW distribution. (G) For $(e, f)=\mathbb{R}, b=1, \lambda=0, \psi(x)=e^{1-(\bar{G}(x ; \eta))^{-\gamma}}$ and $\delta=\frac{\theta}{\gamma+\theta}$, Proposition H1 provides a characterization of Go-G distribution. (H) For $(e, f)=(0, \infty), a=1, \psi(x)=1-\exp \left(-\theta x^{-2}\right)$ and $\delta=\frac{b}{b+1}$, Proposition H1 provides a characterization of BIR distribution. (I) For $(e, f)=(0, \infty), b=1, \psi_{1}(x)=\exp \left(-x^{-2}\right)$ and $\delta_{1}=\frac{a \theta}{a \theta+1}$ , Proposition $\mathrm{H} 2$ provides a characterization of BIR distribution. $(J)$ For $(e, f)=(0, \infty), a=1, \psi(x)=\frac{1-e^{\lambda e^{-\beta_{x}^{\alpha}}}}{1-e^{\lambda}}$ and $\delta=\frac{b}{b+1}$, Proposition H1 provides a characterization of BWP distribution. $(K)$ For $(e, f)=(0, \infty), b=1, \psi_{1}(x)=$ $\frac{e^{\lambda}-e^{\lambda e^{-\beta x^{x}}}}{e^{\lambda}-1}$ and $\delta_{1}=\frac{a}{a+1}$, Proposition $\mathrm{H} 2$ provides a characterization of BWP distribution. $(L)$ For $(e, f)=(0, \infty), a=1$, $\psi(x)=e^{-x^{\beta} e^{\lambda x}}$ and $\delta=\frac{\alpha}{\alpha+1}$, Proposition H1 provides a characterization of GMW distribution. (M) For $(e, f)=(0, \infty)$, $\lambda=0, \psi_{1}(x)=\left(1+\alpha x^{-\theta}\right)^{-1}$ and $\delta_{1}=\frac{\beta}{\beta+1}$, Proposition $\mathrm{H} 2$ provides a characterization of TD distribution. (N) For $(e, f)=\mathbb{R}, \psi_{1}(x)=1-e^{\frac{\theta}{\gamma}\left(1-(\bar{G}(x ; \eta))^{-\gamma}\right)}$ and $\delta_{1}=\frac{\alpha}{\alpha+1}$, Proposition H2 provides a characterization of EGG distribution. $(O)$ For $(e, f)=(0, \infty), \alpha=1, \psi(x)=\left(1+\frac{\lambda}{1+\lambda} x^{\beta}\right)^{1 / \lambda} e^{-x^{\beta}}$ and $\delta=\frac{\lambda}{\lambda+1}$, Proposition H1 provides a characterization of NEPL distribution. (P) For $(e, f)=(0, \infty), \psi(x)=1-\frac{\gamma_{1}\left[k,\left(\frac{x}{\alpha}\right)^{T}\right]}{1-p\left\{1-\gamma_{1}\left[k,\left(\frac{x}{\alpha}\right)^{T}\right]\right\}}$ and $\delta=\frac{\lambda}{\lambda+1}$, Proposition H1 provides a characterization of EGGG distribution. ( $Q$ ) For $(e, f)=(0, \infty), \lambda=0, \beta=1, \psi(x)=\left(\frac{\mu \theta+1+\theta x}{\mu \theta+1} e^{-\theta x}\right)$ and $\delta=\frac{\alpha}{\alpha+1}$ , Proposition H1 provides a characterization of ETQL distribution. $(R)$ For $(e, f)=(0, \infty), \psi(x)=\left(\frac{\alpha+\beta+\alpha \beta x}{\alpha+\beta}\right)^{1 / \alpha} e^{-x}$ and $\delta=\frac{\alpha}{\alpha+1}$, Proposition H1 provides a characterization of AEE distribution. $(S)$ For $(e, f)=(0,1), \psi(x)=$ $\exp \left[-\left(\frac{x}{1-x}\right)^{\beta}\right]$ and $\delta=\frac{1}{2}$, Proposition H1 provides a characterization of ERK distribution. $(T)$ For $(e, f)=(0, \infty)$, $\psi(x)=\left(\frac{\alpha+\beta+\alpha \beta x}{\alpha+\beta}\right)^{1 / \alpha} e^{-x}$ and $\delta=\frac{\alpha}{\alpha+1}$, Proposition H1 provides a characterization of NEE distribution. $(U)$ For $(e, f)=(0, \infty), \psi_{1}(x)=1-e^{1-(1+\alpha x)^{\beta}}$ and $\delta=\frac{\lambda}{\lambda+1}$, Proposition $\mathrm{H} 2$ provides a characterization of ENHE distribution. (V) For $(e, f)=\mathbb{R}, \psi(x)=\left(\exp \left\{-[-\ln (\bar{G}(x ; \eta))]^{b}\right\}\right)^{a}$ and $\delta=\frac{a}{a+1}$, Proposition H1 provides a characterization of GAW-G distribution. (W) For $(e, f)=(0, \infty), \alpha=1, \psi_{1}(x)=1-e^{-\lambda x^{\beta}}$ and $\delta_{1}=\frac{v+1}{v+2}$, Proposition H2 provides a characterization of Gww distribution. $(X)$ For $(e, f)=(0, \infty), \psi(x)=e^{-(x / \theta)^{\lambda}}\left(3-2 e^{-(x / \theta)^{\lambda}}\right)^{1 / 2}$ and $\delta=\frac{2}{3}$, Proposition $\mathrm{H} 1$ provides a characterization of GB distribution.

\section{Infinite Divisibility}

Bondesson (1979) showed that all the members of the following families

$$
\begin{gathered}
f(x)=C x^{\beta-1}\left(1+c x^{\alpha}\right)^{-\gamma}, \quad x>0, \quad 0<\alpha \leq 1, \\
f(x)=C x^{\beta-1} \exp \left\{-c x^{\alpha}\right\}, \quad x>0, \quad 0<|\alpha| \leq 1, \\
f(x)=C x^{\beta-1} \exp \left\{-\left(c_{1} x+c_{2} x^{-1}\right)\right\}, \quad x>0,-\infty<\beta<\infty, \\
f(x)=C x^{-1} \exp \left\{-(\log x-\mu)^{2} /\left(2 \sigma^{2}\right)\right\}, \quad x>0,
\end{gathered}
$$

where the natural restrictions are put on the unspecified parameters, are infinitely divisible. The last one is the lognormal density.

Remark 3.1. Bondesson (1992, Theorem 6.2.4) pointed out that multiplying densities $\left(B_{1}\right)-\left(B_{4}\right)$ by $C_{1}(\delta+x)^{-v}$ for $\delta>0$ and $v>0$, will result in densities which are also infinitely divisible.

Remark 3.2. The distributions, listed in Section 1, whose densities (or densities of their transformations) can be expressed, in view of Remark 3.1, in the form $\left(B_{2}\right)$ are: EGLFR for $\alpha=1, \beta=b=0$; Kw-TEMW for $\theta=\lambda=0,0<\beta<1$ ; the $p d f$ of $Y=e^{-s X}$, where $X$ has TEE distribution with $a=2, \alpha=1$; BWP for $a=1, \lambda=0$ and $0<\beta \leq 1$; GMW for $a=1, \lambda=0$; AEE $\alpha \leq 1$; NEE for $\alpha \leq 1$. The $p d f$ of $Y=e^{s X}$, where $X$ has LE distribution can be written in the form of $\left(B_{1}\right)$. The $p d f$ of the distribution BIR (for $b=1$ ) can be written in the form of $\left(B_{4}\right)$. 


\section{Concluding Remarks}

In designing a stochastic model for a particular modeling problem, an investigator will be vitally interested to know if their model fits the requirements of a specific underlying probability distribution. To this end, the investigator will vitally depend on the characterizations of the selected distribution. A good number of recently introduced distributions which have important applications in many different fields have been mentioned in this work. Certain characterizations of these distributions have been established. We hope that these results will be of interest to the investigators who may believe their models have distributions mentioned here and are looking for justifying the validity of their models. It is known that determining a distribution is infinitely divisible or not via the existing representations is not easy. We have used Bondesson's classifications to show that some of the distributions taken up in this work are infinitely divisible. This could be helpful to some researchers. Finally, we like to mention that the distributions mentioned in this work may be a source of preventing duplications of so called newly proposed distributions.

\section{References}

Abd-EAbd-Elrahman, A.M. (2017). A new two-parameter lifetime distribution with decreasing, increasing or upsidedown bathtub shaped failure rate. Commun. Statist. Theo. Meth., 46(18), 8865-8880.

Abdul-Moniem, I.B. (2015). Exponentiated Nadarajah and Haghighi exponential distribution. International j.of Mathematical Analysis and Applications (IJMAA), 2(5), 68-73.

Abdul-Moniem, I.B., \& Abdel-Hameed, H.F. (2012). On exponentiated Lomax distribution. International J. of Mathematical Archive (IJMA), 3(5), 2144-2150.

Abouelmagd, T.H.M., Hamed, M.S., \& Ebraheim, A.E.H.N. (2017). The Poisson-G family of distributions with applications. Pak.j.stat.oper.res. XIII(2), 313-326.

Al-babtain, A., Fattah, A.A. Ahmed, A-hadi N., \& Merovci, F. The Kumaraswamy-transmuted exponentiated modified Weibull distribution. (Commun. Statist. Simulation and Computation, forthcoming).

Alizadeh, M., Cordeiro, G.M., Pinho, L.G.B., \& Ghosh, I. (2017). The Gompertz-G family of distributions. J. Statistical Theory and Practice, 11, 179-207.

Alizadeh, M., Emadi, M., Doostparast, M., Cordeiro, G.M., Ortega, E.M.M., \& Pescim, R. R. (2015). A new family of distributions: The Kumaraswamy odd log-logistic. properties and applications, HJMS, 44 1491-1512.

Alizadeh, M., MirMostafaee, S.M.T.K., \& Ghosh, I. (2017). The new extension of power Lindley distribution for analyzing binomial data. Chilean J.of Statistics, 8(1), 67-86.

Aryal, G.R., \& Yousof, H. M. The exponentiated generalized-G Poisson family of distributions. (Stochastics and Quality Control, forthcoming).

Babu, M.G. (2016). On a generalization of Weibull distribution and its applications. International J. of Statistics and Applications, 6(3), 168-176.

Bidram, H., Alamatsaz, M.H., \& Nehoukhou, V. (2015). On an extension of exponentiated Weibull distribution. Commun. Statist. Theo-Meth. 44, 1389-1404.

Bortolini, J., Pascoa, M.A.R., de Lima, R.R., \& Oliveira, A.C.S. (2017). The extended generalized gamma geometric distribution. IJSP, 6(4), 48-69.

Chung, Y., Dey, D.K., \& Jung, M. (2017). The exponentiated inverse Weibull geometric distribution. PJS, 33(3), 161-178.

Cordeiro, G.M., Alizadeh, M., Nascimento, A.D.C., \& Rasekhi, M. (2016). The exponentiated Gompertz family of distributions: properties and applications. Chilean J.of Statistics, 7(2), 29-50.

Cordeiro, G.M., Alizadeh, M., \& Ortega, E.M.M., The exponentiated half-logistic family of distributions: properties and applications. J. of Probability and Statistics, Article ID 864396: 1-21.

Cordeiro, G.M., Alizadeh, M. and Pinho, L.G.B. (2016). The type I half-logistics family of distributions. J. Statistical Computation and Simulation, 86, 707-728.

Cordeiro, G.M., Aristizábal, W.D., Suárez, D.M., \& Lozano, S. (2015). The gamma modified Weibull distribution. Chilean J.of Statistics, 6(1), 37-48.

da Silva Braga, A., Cordeiro, G.M., \& Ortega, E.M.M., A new skew-bimodal distribution with applications. (Commun. Statist. Theo-Meth. forthcoming).

Elbatal, I., \& Aryal, G. (2015). Transmuted Dagum distribution with applications. Chilean J.of Statistics, 6(2), 31-45. 
Elbatal, I., El Gebaly, Y.M., \& Amin, E.A. (2017).The beta generalized inverse Weibull geometric distribution and its applications, Pak.j.stat.oper.res. XIII(1), 75-90.

Elbatal, I., Mansour, M.M., \& Ahsanullah, M. (2016). The additive Weibull-geometric distribution: theory and applications. JSTA, 15, 125-141.

Glänzel, W. A characterization theorem based on truncated moments and its application to some distribution families, Mathematical Statistics and Probability Theory (Bad Tatzmannsdorf, 1986). Vol. B, Reidel, Dordrecht, 1987, 7584.

Glänzel, W. (1990). Some consequences of a characterization theorem based on truncated moments. Statistics: A Journal of Theoretical and Applied Statistics, 21(4),613-618.

Glänzel, W., \& Hamedani, G.G. (2001). Characterizations of univariate continuous distributions. Studia Sci. Math. Hungar., 37,83-118.

Gómez, Y.M., Bolfarine, H., \& Gómez, H.W. (2014). A new extension of the exponential distribution. Revista Colombiana de Estadistica 37(1), 25-34.

Gomes, A.E., Da-Silva, C.Q., \& Cordeiro, G.M. (2015). The exponentiated G Poisson model. Commun. Statist. TheoMeth 44 4217-4240.

Hamedani, G.G. (2016). On characterizations and infinite divisibility of recently introduced distribution. SMH, 53(4), 467-511.

Hamedani, G.G. (2013). On certain generalized gamma convolution distributions II, Technical Report No. 484, MSCS, Marquette University.

Hamedani, G.G. (2017). Characterizations and infinite divisibility of certain 2016-2017 univariate continuous distributions II. IMF (International Mathematical Forum). 12(12), 565-609.

Hamedani, G.G., \& Safavimanesh, F. (2017). Characterizations and infinite divisibility of certain 2016 univariate continuous distributions. IMF, $12(5), 195-228$.

Hami Golzar, N., Ganji, M., \& Bevrani, H. (2017). The Lomax-exponential distribution, some properties and applications, JSRI (J. of Statistical Research of Iran), 13, 131-153.

Hassan, A.S., Elgarhy, M., \& Shakil, M. (2017). Type II half logistic family of distributions with applications. Pak.j.stat.oper.res. $\mathrm{XIII}(2), 245-264$.

Hassan, A.S., Hameed, S.E., Maiti, S.S., \& Promanik, S. (2017). The generalized additive Weibull-G family of distributions. IJSP, 6(5), 65-83.

Kazemi, M.R., Jafari, A.A., \& Tahmasebi, S. (2017). An extension of the generalized linear failure rate distribution. Commun. Statist. Theo-Meth 46(16), 7916-7933.

Khan, M.S., \& King, R., (2016). New generalized inverse Weibull distribution for lifetime modeling. Communications for Statistical Applications and Methods, 23, 147-161.

Khan, M.S., King, R., \& Hudson, I.L. (2017), Transmuted new generalized inverse Weibull distribution. Pak.j.stat.oper.res. $\mathrm{XIII}(2), 227-296$.

Kharazmi, O., (2016). Generalized weighted Weibull distribution. J. of Mathematical Extension, 10(3), 89-118.

Kharazmi, O., Mahdavi, A., \& Fathizadeh, M., Generalized weighted exponential distribution. (Commun. Statist. Simulation and Computation, forthcoming).

Kharazmi, O., \& Jabbari, L. (2017). A new weighted exponential distribution and its application to the complete and censored data. Gazi University J. of Science, 30(2), 219-236.

Kumar, C.S., \& Dharmaja, S.H.S. (2017). The exponentiated reduced Kies distribution: properties and applications. Commun. Statist. Theo. Meth., 46(17), 8778-8790.

Leão, J., Saulo, H., Bourguignon, M., Cintra, R., Rêgo, L., \& Cordeiro, G. (2013). On some properties of the beta inverse Rayleigh distribution. Chilean J.of Statistics, 4(2), 111-131.

Mahdavi, A., \& Silva, G.O. (2017). A method o expand family of continuous distributions based on truncated distributions. JSRI, 13, 231-247.

Mead, M.E.,(2015). The Kumaraswamy exponentiated Burr XII distribution and its applications. Pak.j.stat.oper.res. 
$\mathrm{XI}(2), 138-148$.

Najarzadegan, H., Alamatsaz, M.H., \& Hayati, S. (2017). Truncated Weibull-G more flexible and more reliable that beta-G distributions. IJSP, 6(5), online 1-17.

Nofal, Z.M., El Gebaly (2017). The generalized transmuted Weibull distribution for lifetime data. Pak.j.stat.oper.res. $\mathrm{XIII}(2), 355-378$.

Nofal, Z.M., El Gebaly, Y.M., Altun, E., Alizadeh, M., \& Butt, N.S. (2017). The transmuted geometric-Weibull distribution: properties, characterizations and regression models. Pak.j.stat.oper.res. XIII(2), 395-416.

Okorie, I.E., Akpanta, A.C., Ohakwe, J., Chikezie, D.C., Onyemachi, C.U., \& Obi, E.O. (2017). The adjusted log-logistic generalized exponential distribution with application to lifetime data. IJSP, 6(4), 1-15.

Pascoa, M.A.R., de Paiva, C.M.M., Cordeiro, G.M., \& Ortega, E.M.M. (2013). The log-Kumaraswamy generalized gamma regression model with application to chemical dependency data. JDS, $11,781-818$.

Percontini, A., Blas, B., \& Cordeiro, G. (2013). The beta Weibull Poisson distribution. Chilean J.of Statistics, 4(2), 3-26.

Preda, V. (2017). Exponentiated transmuted quasi Lindley distribution. IJRT, 7(2), 25-31.

Roozegar, R., Tahmasebi, S., \& Jafari, A. A. (2017). The McDonald Gompertz distribution: Properties and applications. Commun. Statist. Simulation and Computation, 46(5), 3341-3355.

Shakhatreh, M. K. (2012). A two-parameter of weighted exponential distributions. SPL, 82, 252-261.

Vigas, V. P., Silva, G. O., \& Louzada, F. (2017). The Poisson-Weibull regression model. Chilean J.of Statistics, 8(1), 25-51.

\section{Appendix A}

Theorem 1. Let $(\Omega, \mathcal{F}, \mathbf{P})$ be a given probability space and let $H=[a, b]$ be an interval for some $d<b(a=-\infty, b=\infty$ might as well be allowed. Let $X: \Omega \rightarrow H$ be a continuous random variable with the distribution function $F$ and let $q_{1}$ and $q_{2}$ be two real functions defined on $H$ such that

$$
\mathbf{E}\left[q_{2}(X) \mid X \geq x\right]=\mathbf{E}\left[q_{1}(X) \mid X \geq x\right] \xi(x), \quad x \in H,
$$

is defined with some real function $\eta$. Assume that $q_{1}, q_{2} \in C^{1}(H), \xi \in C^{2}(H)$ and $F$ is twice continuously differentiable and strictly monotone function on the set $H$. Finally, assume that the equation $\xi q_{1}=q_{2}$ has no real solution in the interior of $H$. Then $F$ is uniquely determined by the functions $q_{1}, q_{2}$ and $\xi$, particularly

$$
F(x)=\int_{a}^{x} C\left|\frac{\xi^{\prime}(u)}{\xi(u) q_{1}(u)-q_{2}(u)}\right| \exp (-s(u)) d u,
$$

where the function $s$ is a solution of the differential equation $s^{\prime}=\frac{\xi^{\prime} q_{1}}{\xi q_{1}-q_{2}}$ and $C$ is the normalization constant, such that $\int_{H} d F=1$.

We like to mention that this kind of characterization based on the ratio of truncated moments is stable in the sense of weak convergence (see, Glänzel [2]), in particular, let us assume that there is a sequence $\left\{X_{n}\right\}$ of random variables with distribution functions $\left\{F_{n}\right\}$ such that the functions $q_{1 n}, q_{2 n}$ and $\xi_{n}(n \in \mathbb{N})$ satisfy the conditions of Theorem 1 and let $q_{1 n} \rightarrow q_{1}, q_{2 n} \rightarrow q_{2}$ for some continuously differentiable real functions $q_{1}$ and $q_{2}$. Let, finally, $X$ be a random variable with distribution $F$. Under the condition that $q_{1 n}(X)$ and $q_{2 n}(X)$ are uniformly integrable and the family $\left\{F_{n}\right\}$ is relatively compact, the sequence $X_{n}$ converges to $X$ in distribution if and only if $\xi_{n}$ converges to $\xi$, where

$$
\xi(x)=\frac{E\left[q_{2}(X) \mid X \geq x\right]}{E\left[q_{1}(X) \mid X \geq x\right]} .
$$

This stability theorem makes sure that the convergence of distribution functions is reflected by corresponding convergence of the functions $q_{1}, q_{2}$ and $\xi$, respectively. It guarantees, for instance, the 'convergence' of characterization of the Wald distribution to that of the Lévy-Smirnov distribution if $\alpha \rightarrow \infty$.

A further consequence of the stability property of Theorem 1 is the application of this theorem to special tasks in statistical practice such as the estimation of the parameters of discrete distributions. For such purpose, the functions $q_{1}, q_{2}$ and, specially, $\xi$ should be as simple as possible. Since the function triplet is not uniquely determined it is often possible to choose $\xi$ as a linear function. Therefore, it is worth analyzing some special cases which helps to find new characterizations reflecting the relationship between individual continuous univariate distributions and appropriate in other areas of statistics. 


\section{Copyrights}

Copyright for this article is retained by the author(s), with first publication rights granted to the journal.

This is an open-access article distributed under the terms and conditions of the Creative Commons Attribution license (http://creativecommons.org/licenses/by/4.0/). 